\title{
Thermodynamic mixing properties of olivine derived from lattice vibrations
}

\author{
Michel Henricus Gerardus Jacobs • \\ Bernard H. W. S. de Jong
}

Received: 15 March 2008/Accepted: 15 December 2008/Published online: 8 January 2009

(C) The Author(s) 2009. This article is published with open access at Springerlink.com

\begin{abstract}
We use a lattice vibrational technique to derive thermophysical and thermochemical properties of fayalite, $\mathrm{Fe}_{2} \mathrm{SiO}_{4}$. This semi-empirical technique is based on an extension of Kieffer's model to incorporate details of the phonon spectrum. It includes treatment of intrinsic anharmonicity and electronic effects based on crystal field theory. We extend it to predict thermodynamic mixing properties of olivine $(\mathrm{Mg}, \mathrm{Fe})_{2} \mathrm{SiO}_{4}$ solid solutions by using results of our previous work on the system $\mathrm{MgO}-\mathrm{SiO}_{2}$. Achieving this requires a relation between phonon frequency and composition and a composition relation for the energy of the static lattice. Directed by experimental Raman spectroscopic data for specific optic modes in magnesium-iron solid solutions of olivine and pyroxene we use an empirical relation for the composition dependence for phonon frequencies. We show that lattice vibrations have a large effect on the excess entropy and that the static lattice contribution and lattice vibrations have a large impact on excess enthalpy and excess Gibbs energy. Our model indicates that compositional effects in electronic and magnetic properties are negligible. The compositional variation the Néel temperature has a large impact on excess heat capacity for temperatures below $100 \mathrm{~K}$.
\end{abstract}

\footnotetext{
M. H. G. Jacobs ( $\square)$

Department of Theoretical Geophysics, Faculty of Geosciences, Utrecht University, Budapestlaan 4, 3584 CD Utrecht,

The Netherlands

e-mail: jacobs@geo.uu.nl

B. H. W. S. de Jong

Petrology Group, Faculty of Geosciences, Utrecht University,

Budapestlaan 4, 3584 CD Utrecht, The Netherlands
}

Keywords Excess properties - Anharmonicity · Equation of state $\cdot$ Crystal field $\cdot$ Lattice vibrations

\section{Introduction}

Matching acoustic velocities with material properties of crust and mantle is the principal goal in the study of the solid Earth. These material properties are increasingly more determined by detailed experimentation at elevated temperature and pressure. They place constraints on phase transitions, their Clapeyron slopes, and acoustic speeds, thus enabling a precise characterization of the constitution of crust and mantle. The $\mathrm{MgO}-\mathrm{SiO}_{2}$ system with some admixture of $\mathrm{FeO}$ is the canonical system thought to describe the earth crust and mantle as inferred from the study of chronditic meterorites. To go from inference to unequivocal determination requires a detailed analysis of the physical properties of the phases present in this system.

In an earlier study (Jacobs and de Jong 2007) we have shown that multiple-phase transitions in the system $\mathrm{MgO}$ $\mathrm{SiO}_{2}$, at pressure-temperature conditions prevailing in the transition zone of Earth's mantle, are visible in sound wave velocities commensurate with a recent study of Deuss et al. (2006) based on global seismic observations. However, sound wave velocities and densities calculated along plausible adiabatic paths do not match those of PREM (Dziewonski and Anderson 1981) within tomographic accuracy, indicating that at least iron is lacking in the model description. Therefore, our current goal is to include magnesium-iron silicate solid solution phases in our thermodynamic description.

In the present work we focus on the application of the vibrational model to the olivine $(\mathrm{Mg}, \mathrm{Fe})_{2} \mathrm{SiO}_{4}$ solid solution phase, a major constituent material in Earth's transition 
zone and upper mantle, for which thermophysical properties and thermodynamic mixing data are available to validate the model description. We have achieved a thermodynamic description for this phase in two steps. In the first step, we applied the vibrational technique to the endmember fayalite, $\alpha-\mathrm{Fe}_{2} \mathrm{SiO}_{4}$. In the second step, we combined the results with our previous description for forsterite $\alpha-\mathrm{Mg}_{2} \mathrm{SiO}_{4}$ (Jacobs and de Jong 2005a, 2007) to derive thermodynamic mixing properties of olivine, $\alpha-\left(\mathrm{Mg}_{1-x}, \mathrm{Fe}_{x}\right)_{2} \mathrm{SiO}_{4}$. We realize this by using available Raman and infrared spectroscopic data for the compositional dependence of phonon frequencies. Figure 1 illustrates that these frequencies depend nearly linear on the composition for a number of solid solution phases. According to Huang et al. (2000) and Mernagh and Hoatson (1997) a similar trend is present in pyroxenes and according to Hofmeister and Mao (2001) in silicates with the spinel structure.

Guyot et al. (1986) have shown that a quantitative theoretical interpretation of the substitutional effect of magnesium by iron is not straightforward. We follow an empirical approach to describe the phonon frequencies as function of composition. By applying the frequencycomposition relation we predict the vibrational contribution to thermodynamic mixing properties for the excess Gibbs energy. For the static lattice contribution to the mixing properties we took the Helmholtz energy to be a linear combination of the Helmholtz energies for the endmembers at the volume of the mixture. Here, we shall explore the behavior of excess properties in $P-T$ space and investigate if the results are significantly different from those derived by Jacobs and de Jong (2005b) where excess properties were parameterized using polynomial functions.
Two new databases meeting similar requirements have been developed recently: one by Piazzoni et al. (2007) the other by Stixrude and Lithgow-Bertelloni (2005). Thermophysical properties including shear moduli and phase diagrams may be obtained from them. Both data bases use a Debye model to calculate the thermal pressure, but polynomial expressions for thermal expansivity and heat capacity are used in the database of Piazonni et al. (2007). For the shear modulus a formalism developed by Stixrude and Lithgow-Bertelloni (2005) is used. The database of the last investigators combines a Debye model for the vibrational density of states (VDoS) with an advanced model for calculating elastic moduli.

There are four differences between our formalism and that employed by Piazzoni et al. (2007) and Stixrude and Lithgow-Bertelloni (2005). First our formalism incorporates more details of the phonon spectrum. Such details are essential because we noticed the insufficient accuracy of the Debye model to represent heat capacity data from $0 \mathrm{~K}$ up to the melting point in our thermodynamic analysis of forsterite (Jacobs and de Jong 2005a). It compelled us to employ Kieffer's (1979) model to describe experimental data associated with the VDoS, such as frequency-pressuretemperature measurements derived from Raman and infrared spectroscopy. In Jacobs and de Jong (2007) we noticed that Kieffer's model is also required for other minerals, such as for $\mathrm{MgSiO}_{3}$ perovskite. Infrared and Raman experimental data, which constrain our formalism, have become increasingly available through the work of among others Hofmeister and Ito (1992), Wang et al. (1992), Chopelas (2000) and Chaplot et al. (2002). Second, our vibrational technique incorporates intrinsic mode anharmonicity. Experimental data on mode anharmonicity are scant. They
Fig. 1 Vibrational frequency of modes at $300 \mathrm{~K}$ and $1 \mathrm{bar}$ pressure calculated using Eq. 13, solid curves are not significantly different from a linear behavior (dashed curves). Experimental data for olivine are from Guyot et al. (1986), circle, Besson et al. (1982), triangle. Data for pyroxene are from Huang et al. (2000): synthetic crystals: circle, natural specimens, triangle, Chopelas (1999), square
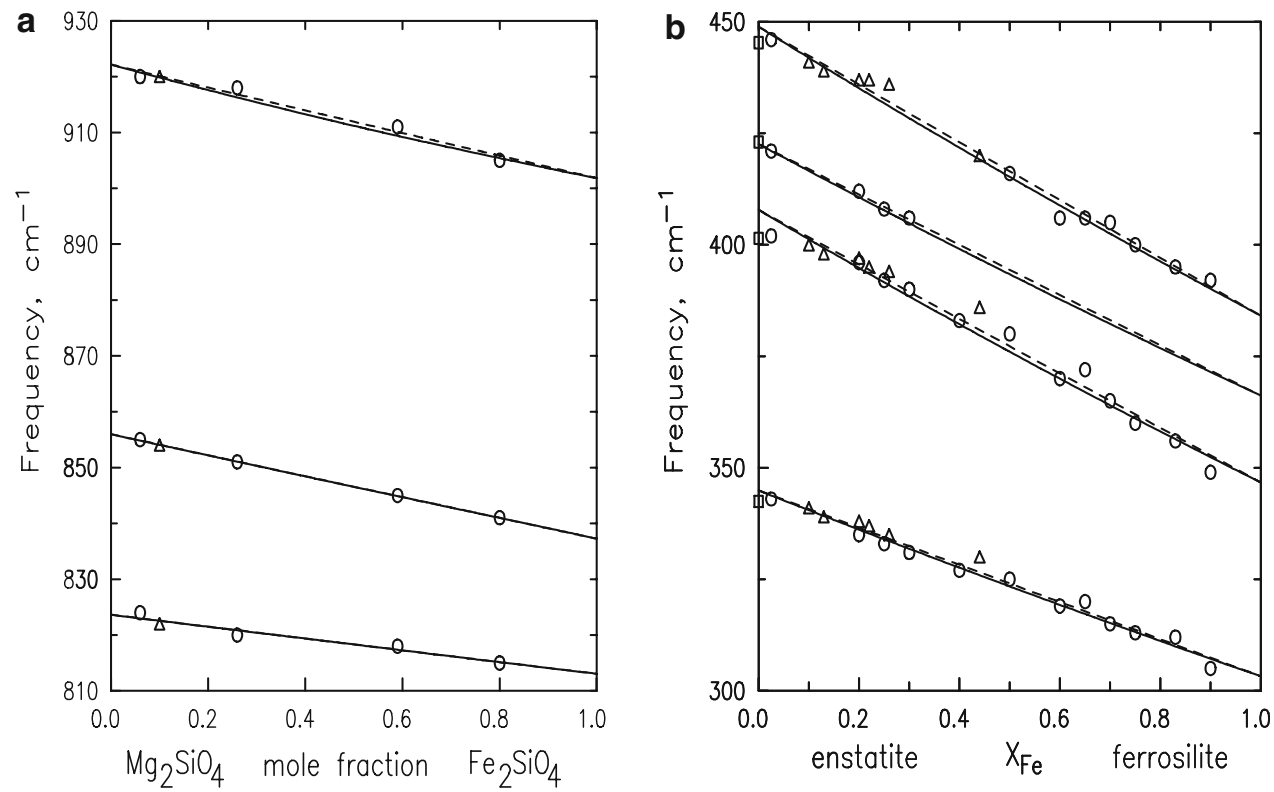
have become available for a number of minerals, such as forsterite, perovskite and akimotoite through the work of Gillet et al. (1991), Gillet et al. (2000) and Reynard and Rubie (1996), respectively. Incorporation of mode anharmonicity is important because as demonstrated in Jacobs and de Jong (2005a) and Jacobs and de Jong (2007), it significantly affects the location and Clapeyron slope of phase boundaries in the magnesium-olivine and magnesium-pyroxene system. Third, we have constructed our formalism such that static lattice properties at $0 \mathrm{~K}$ are key properties, which can be constrained by or compared with $0 \mathrm{~K}$ static ab initio calculations, thus enabling validation of calculation-based properties with experimentally determined ones. This is a profound achievement because it couples molecular calculations, the microscopic world with thermodynamic macroscopic observables. The fourth difference is addressed in this paper in the construction of the thermodynamic model for fayalite. Contrary to forsterite, a model for fayalite, i.e., consideration of iron in the olivine structure requires details of the electronic and magnetic properties to be incorporated. These details involve changes in the thermodynamic properties associated with a change in magnetic ordering at the antiferromagnetic-paramagnetic transition. To model these properties we have used crystal field theory to derive electronic and magnetic properties. An empirical model incorporating the change of magnetic ordering has been included as well.

Because our vibrational model incorporates more physical properties relative to polynomial models used by, e.g., Fabrichnaya (1998), Holland and Powell (1998), Saxena (1996) and used in our previous work Jacobs and de Jong (2005b), the thermodynamic description of substances is more unambiguously constrained. For instance, in Jacobs et al. (2006) we have shown for $\gamma-\mathrm{Mg}_{2} \mathrm{SiO}_{4}$ that this results in a better discrimination of the quality of different experimental data sets. Another example is $\mathrm{MgSiO}_{3}$ perovskite for which a description based on lattice vibrations results in a more reliable extrapolation of thermodynamic properties to regions in pressure-temperature space, which are difficult to access experimentally. These details in our model make a convincing case for the constitution of the crust and upper mantle.

This paper is structured as follows: in the next section we give a brief theoretical background of the vibrational method applied to solid solutions. In "Results", we present our results for fayalite and olivine. In "Discussion" we discuss our results.

\section{Theoretical background}

In Jacobs and de Jong (2007) we applied a vibrational method to the endmembers in the system $\mathrm{MgO}-\mathrm{SiO}_{2}$. The fitting parameters in this method are thermophysical properties obtained by a least-squares optimization. Because we have discussed the method in detail in previous work, Jacobs and de Jong (2005a, 2006, 2007), we only briefly discuss these properties in Appendix 1. Contrary to the endmembers in $\mathrm{MgO}-\mathrm{SiO}_{2}$, electronic and magnetic effects are important in $\mathrm{Fe}_{2} \mathrm{SiO}_{4}$ (fayalite) and we treat these effects in "Electronic and magnetic contributions to the Helmholtz energy in $\mathrm{Fe}_{2} \mathrm{SiO}_{4}$ ". In "Static lattice and vibrational contribution to the Helmholtz energy for a mixture" and "Relation between vibrational frequencies and composition" we extend our vibrational method to a solid solution phase for which the Helmholtz and Gibbs energy are composition dependent.

Electronic and magnetic contributions to the Helmholtz energy in $\mathrm{Fe}_{2} \mathrm{SiO}_{4}$

Our thermodynamic framework is based on the expression of the Helmholtz energy, from which all thermodynamic properties can be derived including the equation of state and the Gibbs energy. Because electronic and magnetic effects are present in $\mathrm{Fe}_{2} \mathrm{SiO}_{4}$, the Helmholtz energy expression given in Appendix 1 is extended to

$$
\begin{aligned}
A(T, V)= & U^{\mathrm{ref}}\left(V_{0}^{\mathrm{st}}\right)+U^{\mathrm{st}}(V)+A^{\mathrm{vib}}(T, V)+A^{\mathrm{el}-\mathrm{mg}}(T, V) \\
& +A^{\lambda}(T, V)
\end{aligned}
$$

The fourth term on the right-hand side of Eq. 1 represents the electronic and magnetic contributions. The last term represents the change in Helmholtz energy due to the antiferromagnetic-paramagnetic transition.

In a recent investigation Aronson et al. (2007) used inelastic neutron scattering experiments to determine the magnetic and electronic contributions to the heat capacity of fayalite. They concluded that the behavior of the M1 site is responsible for the Schottky anomaly appearing in the heat capacity at around $20 \mathrm{~K}$ whereas the M2 site contributes to the lambda behavior in the heat capacity. We followed their crystal-field scheme in which the $T_{2 \mathrm{~g}}$ energy levels are split into a ground state and two energy levels $\delta_{1}$ and $\delta_{2}$, respectively, above it. Spin-orbit coupling further splits the ground state. For completeness we have included the $E_{\mathrm{g}}$ energy levels although their contribution to the heat capacity is less than $0.1 \%$ at $1,400 \mathrm{~K}$. The partition function $Z^{\mathrm{el}-\mathrm{mg}}$ for a system with $m$ energy levels is given by

$Z^{\mathrm{el}-\mathrm{mg}}=\sum_{i=1}^{m} g_{i} \exp \left(\frac{\varepsilon_{i}}{k T}\right)$

where $\varepsilon_{i}$ represents the energy of level $i$ having a degeneracy of $g_{i}$. The Helmholtz energy contribution is derived from it 
by using statistical mechanics as $A^{\mathrm{el}-\mathrm{mg}}=-k T \ln \left(Z^{\mathrm{el}-\mathrm{mg}}\right)$. From Eq. 2 electronic and magnetic contributions to thermodynamic properties are derived using classical thermodynamics. A summary of the expression for these properties is given in Appendix 2. In our calculations we assume that the number of M1 sites equals the number of M2 sites and that there is no site preference of the $\mathrm{Fe}^{2+}$ ion for one of these sites. This is commensurate with a study of Burns and Sung (1978) concluding that the ambient crystal field stabilization enthalpies for the octahedra in the M1 and M2 sites differ by less than 2\%. The assumption also enhances a direct comparison with results derived by Aronson et al. (2007). Because two moles of $\mathrm{Fe}$ atoms per molecular formula of $\mathrm{Fe}_{2} \mathrm{SiO}_{4}$ are present we add the contributions of the thermodynamic properties for each site.

The sharp critical lambda phenomenon in the heat capacity at the Néel temperature $(64.88 \mathrm{~K})$ corresponds to the change in the ordering of the electronic spins when fayalite changes from the antiferromagnetic state to the paramagnetic state with increasing temperature. This phenomenon cannot be described by Eq. 2. We modeled it by making use of an expression for the Gibbs energy compiled by Dinsdale (1991), which is frequently used in the Scientific Group Thermodata Europe (SGTE, http://www. sgte.org) community. This expression only depends on the temperature and we used it to express the Helmholtz energy as

$A^{\lambda}(T)=$ const $\times n_{\mathrm{Fe}} \times R T(g(\tau)-1)$

where $n_{\mathrm{Fe}}$ denotes the number of $\mathrm{Fe}$ atoms per molecular formula $\mathrm{Fe}_{2} \mathrm{SiO}_{4}, n_{\mathrm{Fe}}=2, R$ the gas constant and $\tau=T / T_{\mathrm{N}}$, with $T_{\mathrm{N}}$ the Néel temperature. In Eq. 3 we have assumed that the Helmholtz energy contribution is independent of volume. In "Effect of volume on electronic and magnetic properties of fayalite" we demonstrate that the effect of volume on the Helmholtz energy is quite small. It follows from this assumption that the magnetic contribution to the Gibbs energy equals that for the Helmholtz energy. The function $g(\tau)$ is expressed as

$$
\begin{aligned}
& g(\tau)=1-\left[\frac{0.86034}{\tau}+1.04695\left(\frac{\tau^{3}}{6}+\frac{\tau^{9}}{135}+\frac{\tau^{15}}{600}\right)\right] \\
& \text { for } \tau \leq 1 \\
& g(\tau)=-0.42690\left[\frac{\tau^{-5}}{10}+\frac{\tau^{-15}}{315}+\frac{\tau^{-25}}{1,500}\right] \quad \text { for } \tau>1
\end{aligned}
$$

The constant 'const' results from an optimization process as discussed in "Fayalite". Equation 3 results in zero entropy at $0 \mathrm{~K}$. We do not intend to associate a physical interpretation of the critical lambda effect with Eq. 3, but merely use it as a means to parameterize the energy, entropy and heat capacity.
Static lattice and vibrational contribution to the Helmholtz energy for a mixture

In Jacobs and de Jong (2005b) we used the Gibbs energy to derive element partitioning and phase diagrams for the olivine, wadsleyite and ringwoodite forms of $(\mathrm{Mg}, \mathrm{Fe})_{2} \mathrm{SiO}_{4}$ solid solutions. The Gibbs energy of olivine was expressed on a one-site mixing basis, $(\mathrm{Mg}, \mathrm{Fe}) \mathrm{Si}_{1 / 2} \mathrm{O}_{2}$, as:

$$
\begin{aligned}
G(P, T, \vec{y})= & \sum_{i=1}^{2} y_{i} G_{i}(P, T) \\
& +R T \sum_{i=1}^{2} y_{i} \ln \left(y_{i}\right)+G^{\mathrm{Ex}}(P, T, \vec{y})
\end{aligned}
$$

where $G_{i}(P, T)$ represents a polynomial expression for the Gibbs energy of endmember $i$ typically containing about 16 terms to describe heat capacity, thermal expansivity and bulk modulus. Throughout this paper we denote $\mathrm{MgSi}_{1 / 2} \mathrm{O}_{2}$ as the first endmember and $\mathrm{FeSi}_{1 / 2} \mathrm{O}_{2}$ as the second one. In Eq. 5 we use $\vec{y}=\left(y_{1}, y_{2}\right)$ to represent the composition of the mixture to avoid confusion with the variable $x$ related to vibrational frequencies which we used in previous work and in the appendices. The first term in Eq. 5 represents the Gibbs energy of the so-called mechanical mixture in which the endmembers are present in an unmixed state. The second term represents the Gibbs energy contribution due to random (ideal) mixing of the $\mathrm{Mg}$ and $\mathrm{Fe}$ atoms. The last term is the excess Gibbs energy representing the deviation from ideal mixing of these atoms, which is expressed as a polynomial function in composition in Jacobs and de Jong (2005b). In the present work, we attempt to partition this contribution due to changes in the static lattice and lattice vibrations when mixing occurs.

We showed previously (Jacobs and de Jong 2007) the advantage of using the Helmholtz energy in expressing the Gibbs energy of the endmembers in terms of lattice vibrations. The reason for this is that the Helmholtz energy is linked to the partition function and amenable to statistical mechanical treatment. From the Helmholtz energy we derived the equation of state, partitioned in a static lattice and a vibrational part as is summarized in Appendix 1 for pure endmembers. That results in the volume of the static lattice, which enables the comparison of our results with static ab initio results as was done for perovskite in Jacobs et al. (2006). Rewriting Eq. 5 in terms of the Helmholtz energy leads to

$$
\begin{aligned}
G(P, T, \vec{y})= & \sum_{i=1}^{2} y_{i} A_{i}\left(T, V_{i}\right)+R T \sum_{i=1}^{2} y_{i} \ln \left(y_{i}\right) \\
& +A^{\operatorname{Ex}}(T, V, \vec{y})+P V
\end{aligned}
$$

where $V_{i}$ in the first term, describing the mechanical contribution, denotes the volume of endmember $i$ at $(P, T)$. 
The excess Helmholtz energy of the mixture represents the change in Helmholtz energy resulting from changes of vibrational frequencies, the volume change due to the difference in size of the $\mathrm{Mg}$ and $\mathrm{Fe}$ atoms, and electronic and magnetic effects relative to the endmembers in the unmixed state. The Helmholtz contribution of the static lattice depends only on the volume and we write the expression for the Helmholtz energy of the mixture as

$$
\begin{aligned}
A(T, V, \vec{y})= & \sum_{i=1}^{2} y_{i} A_{i}^{\mathrm{st}}\left(V_{i}\right)+\sum_{i=1}^{2} y_{i} A_{i}^{\mathrm{vib}}\left(T, V_{i}\right) \\
& +\sum_{i=1}^{2} y_{i} A_{i}^{\mathrm{el}-\mathrm{mg}}(T)+A^{\lambda}(T, \vec{y}) \\
& +R T \sum_{i=1}^{2} y_{i} \ln \left(y_{i}\right)+A^{\prime \mathrm{Ex}}(T, V, \vec{y})
\end{aligned}
$$

Two restrictions are associated with Eq. 7. The first restriction is that we have assumed that the Helmholtz energy contributions of the three effects can be partitioned in an additive manner. The second one is that for olivine no experimental data are available for volume- and compositional effects on the electronic and magnetic contributions to the Helmholtz energy. We therefore assume that these contributions are solely temperature dependent. The linear composition dependence of the third term as a consequence does not contribute to the excess Helmholtz energy. In "Effect of volume on electronic and magnetic properties of fayalite" we discuss the effect of volume-dependent $A_{i}^{\mathrm{el}-\mathrm{mg}}$ on thermodynamic properties. Because $\mathrm{Mg}_{2} \mathrm{SiO}_{4}$ is an insulator material, only $\mathrm{Fe}_{2} \mathrm{SiO}_{4}$ $(i=2)$ contributes to this term. For the lambda antiferromagnetic-paramagnetic transition Dachs and Geiger (2007) and Dachs et al. (2007) showed that the Néel temperature depends on composition and therefore $A^{\lambda}$ may contribute to the excess Helmholtz energy. This excess contribution is therefore not included in $A^{\mathrm{Ex}}(T, V, \vec{y})$. The calculation of the excess contribution due to this transition is detailed in Appendix 3.

In "Relation between vibrational frequencies and composition" we introduce a relation between vibrational frequency and composition. We use this relation to replace the second term on the right-hand side of Eq. 7 by $A^{\mathrm{vib}}(T, V, \vec{y})$. This implies the incorporation of a vibrational excess Helmholtz energy expressed as

$A^{\text {Exvib }}(T, V, \vec{y})=A^{\mathrm{vib}}(T, V, \vec{y})-\sum_{i=1}^{2} y_{i} A_{i}^{\mathrm{vib}}\left(T, V_{i}\right)$

In "Olivine solid solutions" we demonstrate that this replacement requires the introduction of an excess contribution to the static lattice part of the Helmholtz energy. Due to substitution of $\mathrm{Mg}$ by $\mathrm{Fe}$, bonds such as $\mathrm{Mg}-\mathrm{O}$ and $\mathrm{Si}-\mathrm{O}$ change in length, but the topology of the structure remains unchanged. Instead of pursuing a microscopic description of the energy changes in these bonds associated with the iron-magnesium substitution, we approach it phenomenologically by treating the solid solution as being composed of the two endmembers that mix at the volume $V$ of the mixture. In Eq. 7 the first term on the right-hand side is replaced by a term expressing the Helmholtz energy of the endmembers at this volume. That results in the incorporation of an excess contribution to the static lattice

$A^{\mathrm{Exst}}(V)=\sum_{i=1}^{2} y_{i}\left(A_{i}^{\mathrm{st}}(V)-A_{i}^{\mathrm{st}}\left(V_{i}\right)\right)$

with the restrictions and assumptions mentioned above, Eq. 7 is rewritten as

$$
\begin{aligned}
A(T, V, \vec{y})= & \sum_{i=1}^{2} y_{i} A_{i}^{\mathrm{st}}(V)+A^{\mathrm{vib}}(T, V, \vec{y})+y_{2} A_{2}^{\mathrm{el}-\mathrm{mg}}(T) \\
& +A^{\lambda}(T, \vec{y})+R T \sum_{i=1}^{2} y_{i} \ln \left(y_{i}\right) \\
& +A^{\prime \prime \mathrm{Ex}}(T, V, \vec{y})
\end{aligned}
$$

In the present work we have put the last term in Eq. 10 to zero and we investigate the effects on excess properties associated with the first, second, and fourth term on the right-hand side of Eq. 10. The volume of the solid solution is derived from the expression for the total pressure, which follows from Eq. 10, by differentiation to volume as

$P=\sum_{i=1}^{2} y_{i} P_{i}^{\mathrm{st}}(V)+P^{\mathrm{vib}}(T, V, \vec{y})$

where $P_{i}^{\text {st }}(V)$ represents the contribution due to the static lattice of component $i$ and $P^{\mathrm{vib}}$ the thermal pressure. The first term in Eq. 11 is calculated using the static lattice properties given in Table 1. The last term in Eq. 11 is predicted using the relation between vibrational frequencies and composition. Appendix 4 gives a summary of the partitioning of excess properties in contributions of the static lattice, lattice vibrations, and electronic and magnetic properties. The volume of the endmembers is calculated with Eq. 22. Excess volume is calculated by computing the volume of the mixture and the volumes of the endmembers at the selected condition of pressure and temperature and applying

$V^{\mathrm{E}}(P, T, \vec{y})=V(P, T, \vec{y})-\sum_{i=1}^{2} y_{i} V_{i}(P, T)$

Relation between vibrational frequencies and composition

Vibrational frequencies decrease for $\left(\mathrm{Mg}_{1-y}, \mathrm{Fe}_{y}\right)_{2} \mathrm{SiO}_{4}$ mixtures with increasing iron content as illustrated in 
Table 1 Optimized properties at zero Kelvin and zero pressure

\begin{tabular}{|c|c|c|c|c|c|c|}
\hline Mode type & $\begin{array}{l}\text { Fraction of } \\
\text { oscillators }\end{array}$ & Motion type & Frequency range $\left(\mathrm{cm}^{-1}\right)$ & $\gamma_{j, 0}$ & $q_{1, j 0}$ & $a_{j} \times 10^{5} \mathrm{~K}^{-1}$ \\
\hline \multicolumn{7}{|l|}{$\alpha-\mathrm{Mg}_{2} \mathrm{SiO}_{4}$} \\
\hline $\mathrm{AC} 1$ & $1 / 84$ & TA & $0.00-101.4(2)$ & $1.05(5)$ & $1.70(90)$ & $-4.94(5)$ \\
\hline $\mathrm{AC} 2$ & $1 / 84$ & TA & $0.00-102.4(2)$ & $1.05(5)$ & $1.70(90)$ & $-4.94(5)$ \\
\hline AC3 & $1 / 84$ & LA & $0.00-172.7(1)$ & $1.66(2)$ & $0.78(30)$ & $-1.55(5)$ \\
\hline OC1 & $2 / 84$ & $\mathrm{~T}\left(\mathrm{SiO}_{4}\right)$ & $106.4(60)-147.6(60)$ & $1.02(4)$ & $2.33(17)$ & 0.00 \\
\hline $\mathrm{OC} 2$ & $7 / 84$ & $\mathrm{~T}\left(\mathrm{SiO}_{4}\right)$ & $167.9(63)-229.1(63)$ & $2.21(9)$ & $0.80(16)$ & 0.00 \\
\hline OC3 & $12 / 84$ & $\mathrm{~T}\left[\mathrm{M}(2) \mathrm{O}_{6}\right]$ & $229.2(30)-355.1(30)$ & $1.76(6)$ & $2.49(5)$ & $-2.31(6)$ \\
\hline $\mathrm{OC} 4$ & $12 / 84$ & $\mathrm{~T}\left[\mathrm{M}(1) \mathrm{O}_{6}\right]$ & $279.7(30)-414.2$ (30) & $1.22(6)$ & $2.49(5)$ & $-1.59(6)$ \\
\hline OC5 & $12 / 84$ & $\mathrm{R}\left(\mathrm{SiO}_{4}\right)$ & $305.8(30)-474.1(30)$ & $1.42(6)$ & $2.49(5)$ & $-0.84(6)$ \\
\hline OC6 & $8 / 84$ & $v_{2}\left(\mathrm{SiO}_{4}\right)$ & 409.7 (34)-510.7 (34) & $0.56(9)$ & $2.49(5)$ & $-2.95(6)$ \\
\hline OC7 & $12 / 84$ & $v_{4}\left(\mathrm{SiO}_{4}\right)$ & $507.0(34)-647.2(34)$ & $0.56(8)$ & $-2.78(6)$ & $-0.84(8)$ \\
\hline OC 8 & $4 / 84$ & $v_{1}\left(\mathrm{SiO}_{4}\right)$ & $841.5(34)-842.5(34)$ & $0.41(9)$ & $-3.83(4)$ & $-0.84(8)$ \\
\hline OC9 & $3 / 84$ & $v_{3}\left(\mathrm{SiO}_{4}\right)$ & $871.5(34)-872.5$ (34) & $0.39(9)$ & $-3.83(4)$ & $-0.84(8)$ \\
\hline OC10 & $4 / 84$ & $v_{3}\left(\mathrm{SiO}_{4}\right)$ & 919.5 (34)-920.5 (34) & $0.32(9)$ & $-3.83(4)$ & $-0.84(8)$ \\
\hline OC11 & $5 / 84$ & $v_{3}\left(\mathrm{SiO}_{4}\right)$ & 978.5 (34)-979.5 (34) & $0.56(9)$ & $-3.83(4)$ & $-0.84(8)$ \\
\hline$K_{0}^{\mathrm{st}} / \mathrm{GPa}$ & $K_{0}^{\text {st }}$ & & $V_{0} / \mathrm{cm}^{3} / \mathrm{mol}$ & $V_{0}^{\mathrm{st}} / \mathrm{cm}^{3} / \mathrm{mol}$ & $U^{\mathrm{ref}} / \mathrm{MJ} / \mathrm{mol}$ & $Z$ \\
\hline $134.8(1)$ & $4.74(3)$ & & $43.476(48)$ & $43.093(48)$ & $-2.2533(40)$ & 4 \\
\hline \multicolumn{7}{|c|}{$\alpha-\mathrm{Fe}_{2} \mathrm{SiO}_{4}$ based on experiments of Graham et al. (1988), model G } \\
\hline $\mathrm{AC} 1$ & $1 / 84$ & TA & $0.00-67.46(20)$ & $1.88(5)$ & $0.05(90)$ & $-7.00(5)$ \\
\hline $\mathrm{AC} 2$ & $1 / 84$ & TA & $0.00-68.46(20)$ & $1.88(5)$ & $0.05(90)$ & $-7.00(5)$ \\
\hline $\mathrm{AC} 3$ & $1 / 84$ & LA & $0.00-133.26(10)$ & $2.12(2)$ & $0.05(30)$ & $-3.95(5)$ \\
\hline OC1 & $2 / 84$ & $\mathrm{~T}\left(\mathrm{SiO}_{4}\right)$ & $100.5(60)-114.3(60)$ & $2.13(4)$ & $4.59(15)$ & 0.00 \\
\hline $\mathrm{OC} 2$ & $7 / 84$ & $\mathrm{~T}\left(\mathrm{SiO}_{4}\right)$ & $114.3(63)-162.9(63)$ & $1.62(9)$ & $4.59(15)$ & 0.00 \\
\hline OC3 & $12 / 84$ & $\mathrm{~T}\left[\mathrm{M}(2) \mathrm{O}_{6}\right]$ & $162.9(30)-244.9(30)$ & $1.27(6)$ & $4.59(15)$ & 0.00 \\
\hline $\mathrm{OC} 4$ & $12 / 84$ & $\mathrm{~T}\left[\mathrm{M}(1) \mathrm{O}_{6}\right]$ & $174.9(30)-304.9(30)$ & $1.11(6)$ & $4.59(15)$ & 0.00 \\
\hline OC5 & $12 / 84$ & $\mathrm{R}\left(\mathrm{SiO}_{4}\right)$ & $244.9(30)-374.9(30)$ & $1.70(6)$ & $4.59(15)$ & 0.00 \\
\hline OC6 & $8 / 84$ & $v_{2}\left(\mathrm{SiO}_{4}\right)$ & 380.9 (34)-477.4 (34) & $0.29(9)$ & $-4.31(5)$ & 0.00 \\
\hline OC7 & $12 / 84$ & $v_{4}\left(\mathrm{SiO}_{4}\right)$ & 480.9 (34)-608.9 (34) & $0.43(8)$ & $-4.31(6)$ & 0.00 \\
\hline OC8 & $4 / 84$ & $v_{1}\left(\mathrm{SiO}_{4}\right)$ & $826.9(34)-827.9$ (34) & $0.42(9)$ & $-4.31(4)$ & 0.00 \\
\hline OC9 & $3 / 84$ & $v_{3}\left(\mathrm{SiO}_{4}\right)$ & 863.9 (34)-864.9 (34) & $0.40(9)$ & $-4.31(4)$ & 0.00 \\
\hline OC10 & $4 / 84$ & $v_{3}\left(\mathrm{SiO}_{4}\right)$ & 890.9 (34)-891.9 (34) & $0.39(9)$ & $-4.31(4)$ & 0.00 \\
\hline OC11 & $5 / 84$ & $v_{3}\left(\mathrm{SiO}_{4}\right)$ & 950.9 (34)-951.9 (34) & $0.62(9)$ & $-4.31(4)$ & 0.00 \\
\hline$K_{0}^{\mathrm{st}} / \mathrm{GPa}$ & $K_{0}^{\prime \text { st }}$ & & $V_{0} / \mathrm{cm}^{3} / \mathrm{mol}$ & $V_{0}^{\mathrm{st}} / \mathrm{cm}^{3} / \mathrm{mol}$ & $U^{\mathrm{ref}} / \mathrm{MJ} / \mathrm{mol}$ & $Z$ \\
\hline $136.3(8)$ & $5.20(3)$ & & $46.035(33)$ & $45.749(33)$ & $-1.5519(14)$ & 4 \\
\hline \multicolumn{7}{|c|}{$\alpha-\mathrm{Fe}_{2} \mathrm{SiO}_{4}$ based on experiments of Isaak (1993), model I } \\
\hline $\mathrm{AC} 1$ & $1 / 84$ & TA & $0.00-67.71(20)$ & $1.91(5)$ & $0.03(90)$ & $-7.00(5)$ \\
\hline $\mathrm{AC} 2$ & $1 / 84$ & TA & $0.00-68.71(20)$ & $1.91(5)$ & $0.03(90)$ & $-7.00(5)$ \\
\hline $\mathrm{AC} 3$ & $1 / 84$ & LA & $0.00-135.47(10)$ & $2.07(2)$ & $0.05(30)$ & $-4.52(5)$ \\
\hline OC1 & $2 / 84$ & $\mathrm{~T}\left(\mathrm{SiO}_{4}\right)$ & $101.1(60)-115.0(60)$ & $2.10(4)$ & $4.62(15)$ & 0.00 \\
\hline $\mathrm{OC} 2$ & $7 / 84$ & $\mathrm{~T}\left(\mathrm{SiO}_{4}\right)$ & $115.0(63)-163.5(63)$ & $1.58(9)$ & $4.62(15)$ & 0.00 \\
\hline OC3 & $12 / 84$ & $\mathrm{~T}\left[\mathrm{M}(2) \mathrm{O}_{6}\right]$ & $163.5(30)-245.5(30)$ & $1.27(6)$ & $4.62(15)$ & 0.00 \\
\hline OC4 & $12 / 84$ & $\mathrm{~T}\left[\mathrm{M}(1) \mathrm{O}_{6}\right]$ & $172.5(30)-302.5(30)$ & $1.12(6)$ & $4.62(15)$ & 0.00 \\
\hline OC5 & $12 / 84$ & $\mathrm{R}\left(\mathrm{SiO}_{4}\right)$ & $245.5(30)-375.5(30)$ & $1.70(6)$ & $4.62(15)$ & 0.00 \\
\hline OC6 & $8 / 84$ & $v_{2}\left(\mathrm{SiO}_{4}\right)$ & 379.7 (34)-476.2 (34) & $0.38(9)$ & $-0.45(5)$ & 0.00 \\
\hline OC7 & $12 / 84$ & $v_{4}\left(\mathrm{SiO}_{4}\right)$ & 478.7 (34)-606.7 (34) & $0.53(8)$ & $-0.45(6)$ & 0.00 \\
\hline OC8 & $4 / 84$ & $v_{1}\left(\mathrm{SiO}_{4}\right)$ & $827.2(34)-828.2(34)$ & $0.51(9)$ & $-0.45(4)$ & 0.00 \\
\hline OC9 & $3 / 84$ & $v_{3}\left(\mathrm{SiO}_{4}\right)$ & $864.3(34)-865.3$ (34) & $0.48(9)$ & $-0.45(4)$ & 0.00 \\
\hline OC10 & $4 / 84$ & $v_{3}\left(\mathrm{SiO}_{4}\right)$ & $891.1(34)-892.1(34)$ & $0.47(9)$ & $-0.45(4)$ & 0.00 \\
\hline
\end{tabular}


Table 1 continued

\begin{tabular}{lllllll}
\hline Mode type & $\begin{array}{l}\text { Fraction of } \\
\text { oscillators }\end{array}$ & Motion type & Frequency range $\left(\mathrm{cm}^{-1}\right)$ & $\gamma_{j, 0}$ & $q_{1, j 0}$ & $a_{j} \times 10^{5} \mathrm{~K}^{-1}$ \\
\hline OC11 & $5 / 84$ & $v_{3}\left(\mathrm{SiO}_{4}\right)$ & $952.1(34)-953.1(34)$ & $0.73(9)$ & $-0.45(4)$ & 0.00 \\
$K_{0}^{\text {st }} / \mathrm{GPa}$ & $K_{0}^{\text {st }}$ & & $V_{0} / \mathrm{cm}^{3} / \mathrm{mol}$ & $V_{0}^{\mathrm{st}} / \mathrm{cm}^{3} / \mathrm{mol}$ & $U^{\mathrm{ref}} / \mathrm{MJ} / \mathrm{mol}$ & $Z$ \\
$143.2(9)$ & $5.00(3)$ & & $46.022(26)$ & $45.727(26)$ & $-1.5518(14)$ & 4 \\
Const $=0.67192$ (Eq. 3) & & & & \\
\hline
\end{tabular}

$K_{0}^{\text {st }}$ represents the isothermal bulk modulus of the static lattice, $K_{0}^{\text {st }}$ its isothermal pressure derivative, $a_{j}$ the anharmonicity, $\gamma_{j}$ the Grüneisen parameter of mode $j$ and $q_{j}$ the mode $q$ parameter in Eqs. $(18,24$ and 25. Numbers in parentheses denote the uncertainty in the last digit(s) Motion types are TA transverse acoustic, $L A$ longitudinal acoustic, $T$ translational, $R$ rotational, $v$ internal $\mathrm{SiO}_{4}$ vibrational. The fraction of oscillators refers to one primitive unit cell in which four molecules are present. Vinet et al.'s (1987) equation of state is used for the static lattice. $\mathrm{AC}$ denotes acoustic and $\mathrm{OC}$ optic continuum

Fig. 1. The frequencies depend on the masses of the vibrating atoms, as may be deduced qualitatively using a simple harmonic oscillator model, which relates the frequency, $v$, to the reduced mass of the oscillator, $\mu$, and its force constant $k$ as $v=(1 / 2 \pi)(k / \mu)^{1 / 2}$. In spite of the simple frequency-composition behavior, Guyot et al. (1986) showed that the interpretation of the substitutional effect of magnesium by iron on $\mathrm{Si}-\mathrm{O}$ band shifts is not straightforward and that it involves a three-body effect, such as $\mathrm{Fe}-\mathrm{O}-\mathrm{Si}$ rather than a two-body effect, such as $\mathrm{Fe}-\mathrm{O}$. Instead of attempting to relate vibrational frequencies to atomic masses and the nature of the atomic interactions we follow an empirical approach to describe the phonon frequencies as function of composition. Figure 1a indicates that for three internal vibrational modes in olivine these frequencies show a near-linear behavior with composition. According to Huang et al. (2000) and Mernagh and Hoatson (1997) such a trend is also present in at least 17 internal- and lattice vibrational modes of pyroxenes and Fig. 1b exemplifies that this is the case for a number of these modes. In our empirical approach we follow Lawson (1947) and write for the compositional dependence of a particular vibrational mode

$v(V)=\prod_{i=1}^{n} v_{i}^{y_{i}}(V)$

This expression has the advantage that the Grüneisen parameter of each mode depends linearly on the composition. Figure 1a shows that frequency data for olivine calculated with Eq. 13 are not significantly different from linearity. That also holds for enstatiteferrosilite and diopside-hedenbergite solid solution series measured by Huang et al. (2000) as exemplified by the vibrational modes in Fig. 1b. According to Guyot et al. (1986) Raman and near-infrared data indicate that for olivine no additional bands appear due to the ironmagnesium substitution. However, according to Chopelas (1991) some lattice modes having $A_{g}$ symmetry in olivine with composition $\left(\mathrm{Mg}_{0.88}, \mathrm{Fe}_{0.12}\right)_{2} \mathrm{SiO}_{4}$ might show signs of two-mode behavior. In this case the two modes, one for each endmember, with the same band assignment are visible in the spectra of the solid solution. Figure $1 \mathrm{~b}$ illustrates that the scatter in experimental data for enstatite-ferrosilite measured by Huang et al. (2000) is about $4 \mathrm{~cm}^{-1}$. We anticipate that a similar uncertainty will be present in future measurements on olivine with different compositions. Assuming such possible scatter reveals that the majority of the vibrational modes for olivine measured by Chopelas (1991) approaches linearity. For 7 out of the 34 measured Raman modes, the deviation from linearity is larger than $4 \mathrm{~cm}^{-1}$ and we may have to modify our present thermodynamic description in the future when more details about the compositional dependence of these modes are collected. In the present work we assume one-mode behavior and the calculation of thermodynamic properties proceeds by connecting the cut-off frequencies of each vibrational mode having the same band assignment for the endmembers $\mathrm{Mg}_{2} \mathrm{SiO}_{4}$ and $\mathrm{Fe}_{2} \mathrm{SiO}_{4}$ using Eq. 13. At a specific composition, pressure and temperature the Grüneisen, mode $q$ and anharmonicity parameter of a particular mode have one unique value. Experimental data for the compositional dependence of the vibrational frequencies in olivine and pyroxenes are limited to $300 \mathrm{~K}$ and 1 bar pressure conditions. In the present work we assume that relation (13) is valid at all pressures and temperatures.

\section{Results}

For the thermodynamic analysis of properties of fayalite we started from the different VDoS representations given by Hofmeister (1987). We deviate from these by replacing each motion assignment by an optical continuum. The internal asymmetric stretch $\mathrm{SiO}_{4}$ motion, $v_{3}$, is represented by three Einstein continua as was also done by Chopelas (1990) for forsterite. Following her mode assignment for forsterite, we describe the translational $\mathrm{T}\left[\mathrm{SiO}_{4}\right]$ mode in 
fayalite with two optic continua resulting in a total amount of 11 optic continua, the same as for forsterite. The pressure dependence of the vibrational frequencies measured by Hofmeister et al. (1989) constrained our calculation of mode Grüneisen and mode $q$ parameters. We used our method described by Jacobs and de Jong (2005a, 2007) as an inversion technique to conduct analyses of thermodynamic, vibrational and sound velocity data aiming at a consistent description of these properties. Because the adiabatic bulk modulus of fayalite measured by Graham et al. (1988) and those by Isaak et al. (1993) deviate from each other by about $10 \mathrm{GPa}$, we performed two analyses directing the optimization to either of these two datasets. Table 1 shows the result of two analyses obtained by a least-squares optimization process. Table 2 indicates the data constraining the optimization and compares the calculated and experimental properties for the two analyses. We investigate the effect of the resulting descriptions of the mixing properties for olivine solid solutions in "Discussion".

\section{Discussion}

In the next two sections we discuss our results for $\mathrm{Fe}_{2} \mathrm{SiO}_{4}$ (fayalite). We combine these results with the results for $\mathrm{Mg}_{2} \mathrm{SiO}_{4}$ and discuss the excess properties and thermophysical properties of the solid solution $\left(\mathrm{Mg}_{1-y}, \mathrm{Fe}_{y}\right)_{2} \mathrm{SiO}_{4}$ in "Olivine solid solutions", "Excess properties at low temperature" and "Sound wave velocities of olivine".

\section{Fayalite}

Figure 2 indicates a difference of about $10 \mathrm{GPa}$ between the adiabatic bulk modulus for fayalite measured by Sumino (1979) vis a vis Graham et al. (1988), static compression measurements resulting in the lowest values for this property. Isaak et al. (1993) performed a detailed analysis of the source for this discrepancy in the acoustic measurements. Their recommended values are based on their own new measurements and those of Sumino (1979), Graham et al. (1988) and Wang et al. (1989). Their conclusion is that the discrepancy arises from the different ways in which the various experimental techniques sample compositional and microstructural heterogeneities in the probed specimen. How these heterogeneities propagate as systematic errors in the different experimental techniques could not be determined precisely.

Because of the much larger uncertainty in the bulk modulus of fayalite vis a vis forsterite, about $1 \mathrm{GPa}$, we performed two analyses. In analysis $G$ we directed the optimization towards the data of Graham et al. (1988) and in analysis I we directed it towards the recommended data of Isaak et al. (1993). Here we investigate the effect of these analyses on the thermophysical properties of fayalite.

We used in both analyses $G$ and I the crystal-field formulation for the electronic and magnetic effects given by Aronson et al. (2007). The energy levels and their degeneracy are given in Table 3. Aronson et al. (2007) computed the contributions of the M2 site to the electronic properties by using a fivefold degenerate ground state together with the energy differences $\delta_{1}$ and $\delta_{2}$ reported by Burns (1985). According to Eq. 2 the expression for the entropy is

$$
\begin{aligned}
S^{\mathrm{el}-\mathrm{mg}}= & k \ln \left(\sum_{i=1}^{n} g_{i} \exp \left(-\frac{\varepsilon_{i}}{k T}\right)\right) \\
& +\frac{1}{T} \frac{\sum_{i=1}^{n} g_{i} \varepsilon_{i} \exp \left(-\varepsilon_{i} / k T\right)}{\sum_{i=1}^{n} g_{i} \exp \left(-\varepsilon_{i} / k T\right)}
\end{aligned}
$$

Fivefold degeneracy in the ground state with $\varepsilon_{1}=0$ leads to a non-zero value for the entropy, $k \ln \left(g_{1}\right)$ at $0 \mathrm{~K}$ and zero pressure. This is corrected for by dividing the partition function by $g_{1}$. This correction only affects the entropy and Helmholtz energy.

The partitioning of the heat capacity and entropy in electronic, magnetic, critical lambda, and lattice vibrational contributions as depicted in Fig. 3 is not trivial. The heat capacity is partitioned as

$C_{\mathrm{P}}=C_{\mathrm{V}}^{\mathrm{el}-\mathrm{mg}}+C_{\mathrm{V}}^{\lambda}+C_{\mathrm{V}}^{\mathrm{vib}}+\alpha^{2} K V T$

where $\alpha$ represents thermal expansivity and $K$ the isothermal bulk modulus. The first term on the right-hand side of Eq. 15 is calculated with the values given in Table 3 and Eq. 36 assuming that the transition energies are independent of volume. Our analysis indicates that the last term in Eq. 15 is about $0.17 \%$ of the total heat capacity at $100 \mathrm{~K}$, which is small compared to $3.7 \%$ of the first term. To derive the heat capacity of the critical lambda effect, Aronson et al. (2007) subtracted the contribution of the first term and the lattice vibrational contribution from the measured heat capacity. They estimated contributions of the last term from values given by Hofmeister (1987). Because the lattice vibrational heat capacity at constant volume is not experimentally measurable, Aronson et al. (2007) used their measured heat capacity at constant pressure of monticellite, $\mathrm{CaMgSiO}_{4}$ and applied a procedure recommended by Robie (1982). Their calculated lattice vibrational contribution represents the heat capacity $\left(C_{P}\right)$ data for $\gamma-\mathrm{Ca}_{2} \mathrm{SiO}_{4}$ measured by King (1957), a substance previously used by Robie (1982) to derive the magnetic contribution to the heat capacity of fayalite. Figure 3 shows that our calculated lattice vibrational contribution deviates from the contribution established by Aronson et al. (2007). For comparison we have plotted the heat capacity of $\mathrm{Mg}_{2} \mathrm{SiO}_{4}$. Because the atomic mass of 
Table 2 Representation of thermodynamic properties and mode frequencies for fayalite using model G and I, respectively

\begin{tabular}{|c|c|c|c|c|c|}
\hline Property & $\begin{array}{l}\text { Maximum absolute } \\
\text { deviation in } \% \mathrm{G}, \mathrm{I}\end{array}$ & $\begin{array}{l}\text { Average absolute } \\
\text { deviation in } \% \mathrm{G}, \mathrm{I}\end{array}$ & $T$-range in $\mathrm{K}$ & $P$-range in $\mathrm{GPa}$ & References \\
\hline \multicolumn{6}{|l|}{ Fayalite } \\
\hline \multirow[t]{9}{*}{ Volume } & $0.29,0.24$ & $0.18,0.13$ & $293-1,173$ & 0.0 & Smyth (1975) \\
\hline & $0.39,0.29$ & $0.19,0.16$ & 296 & $0.0-7.3$ & Yagi et al. (1975) \\
\hline & $0.39,0.46$ & $0.21,0.23$ & 296 & $0.0-4.2$ & Hazen (1977) \\
\hline & $0.33,0.25$ & $0.30,0.22$ & 1,273 & $3.9-6.2$ & Yagi et al. (1987) \\
\hline & $0.39,0.46$ & $0.15,0.20$ & 673 & $1.8-6.2$ & PlymateandStout (1990) \\
\hline & $0.97,1.24$ & $0.58,0.72$ & 298 & $25.4-37.3$ & Williams et al. (1990) \\
\hline & $0.40,0.47$ & $0.22,0.29$ & 298 & $0.1-26.1$ & Andrault et al. (1995) \\
\hline & $0.19,0.08$ & $0.14,0.05$ & 293 & $0.0-9.72$ & Zhang (1998) \\
\hline & $0.65,0.84$ & $0.29,0.27$ & 293 & $0.0-14.0$ & Kudoh and Takeda (1986) \\
\hline \multirow[t]{7}{*}{ Heat capacity } & $8.30,8.20$ & $2.92,2.86$ & $1.4-100$ & 0.0 & Aronson et al. (2007) \\
\hline & $6.88,6.90$ & $1.50,1.48$ & $5-300$ & 0.0 & Dachs et al. (2007) \\
\hline & $8.56,7.99$ & $2.97,2.81$ & $300-1,490$ & 0.0 & Barin (1989) \\
\hline & $9.05,9.07$ & $1.14,1.07$ & $5-381$ & 0.0 & Robie et al. (1982) \\
\hline & $7.63,7.14$ & $3.50,3.29$ & $395-1,406$ & 0.0 & Orr (1953) \\
\hline & $2.97,2.91$ & $0.89,0.92$ & $350-700$ & 0.0 & Watanabe (1982) \\
\hline & $15.60,15.09$ & $6.24,6.39$ & $373-1,473$ & 0.0 & Esser et al. (1933) \\
\hline \multirow[t]{3}{*}{ Enthalpy } & $0.00,0.00$ & $0.00,0.00$ & 298.15 & 0.0 & Robie et al. (1982) \\
\hline & $0.01,0.01$ & $0.006,0.005$ & $0-400$ & 0.0 & Robie et al. (1982) \\
\hline & $0.62,0.58$ & $0.11,0.10$ & $395-1,406$ & 0.0 & Orr (1953) \\
\hline Entropy & $5.70,5.48$ & $0.98,0.90$ & $5-381$ & 0.0 & Robie et al. (1982) \\
\hline Thermal expansivity & $6.48,7.73$ & $2.43,3.13$ & $323-1,098$ & 0.0 & Suzuki (1981) \\
\hline \multirow[t]{4}{*}{$K_{\mathrm{S}}$} & $0.99,5.28$ & $0.86,5.14$ & $273-313$ & 0.0 & Graham et al. (1988) \\
\hline & $7.22,3.36$ & $6.89,2.95$ & $293-673$ & 0.0 & Sumino (1979) \\
\hline & $2.40,1.75$ & $2.40,1.75$ & 298 & 0.0 & Wang et al. (1989) \\
\hline & $3.74,0.45$ & $3.67,0.43$ & $300-500$ & 0.0 & Isaak et al. (1993) \\
\hline \multirow[t]{4}{*}{$v_{\mathrm{L}}$} & $0.21,1.72$ & $0.16,1.68$ & $273-313$ & 0.0 & Graham et al. (1988) \\
\hline & $0.16,1.69$ & $0.15,1.52$ & 298 & $0.0-1.0$ & Graham et al. (1988) \\
\hline & $3.35,2.04$ & $2.68,1.26$ & $293-673$ & 0.0 & Sumino (1979) \\
\hline & $1.40,0.10$ & $1.38,0.08$ & $300-500$ & 0.0 & Isaak et al. (1993) \\
\hline \multirow[t]{4}{*}{$v_{\mathrm{T}}$} & $0.18,0.65$ & $0.18,0.62$ & $273-313$ & 0.0 & Graham et al. (1988) \\
\hline & $0.11,0.55$ & $0.09,0.44$ & 298 & $0.0-1.0$ & Graham et al. (1988) \\
\hline & $2.11,2.11$ & $0.88,0.82$ & $273-673$ & 0.0 & Sumino (1979) \\
\hline & $0.14,0.20$ & $0.13,0.17$ & $300-500$ & 0.0 & Isaak et al. (1993) \\
\hline \multirow[t]{4}{*}{$v_{\mathrm{B}}$} & $0.22,2.30$ & $0.15,2.23$ & $273-313$ & 0.0 & Graham et al. (1988) \\
\hline & $0.23,2.27$ & $0.21,2.06$ & 298 & $0.0-1.0$ & Graham et al. (1988) \\
\hline & $3.96,2.01$ & $3.60,1.62$ & $273-673$ & 0.0 & Sumino (1979) \\
\hline & $2.04,0.04$ & $2.01,0.03$ & $300-500$ & 0.0 & Isaak et al. (1993) \\
\hline Mode frequencies & $2.00,2.00$ & $0.53,0.52$ & 293 & $0.0-42.5$ & Hofmeister (1989) \\
\hline
\end{tabular}

Longitudinal, transverse and bulk sound velocity are represented by $v_{\mathrm{L}}, v_{\mathrm{T}}$, and $v_{\mathrm{B}}$

$\mathrm{Mg}$ is smaller than that of $\mathrm{Fe}$ and $\mathrm{Ca}$, the vibrational frequencies of $\mathrm{Mg}_{2} \mathrm{SiO}_{4}$ are expected to be larger in accordance with Fig. 1. As a consequence the heat capacity values for $\mathrm{Mg}_{2} \mathrm{SiO}_{4}$ are smaller than those for $\mathrm{Fe}_{2} \mathrm{SiO}_{4}$. The difference between our calculated heat capacity of $\mathrm{Fe}_{2} \mathrm{SiO}_{4}$ and that for $\gamma-\mathrm{Ca}_{2} \mathrm{SiO}_{4}$ would be in line with these considerations.
In our analysis we followed a different approach involving two steps. In the first step we optimized the properties in Table 1 by using all experimental data except the heat capacity data below $300 \mathrm{~K}$. We fixed the description for the contribution $C_{\mathrm{V}}^{\mathrm{el}-\mathrm{mg}}$ by using the properties given in Table 3. The result of this step is that all terms except the second term in Eq. 15 are known as a 


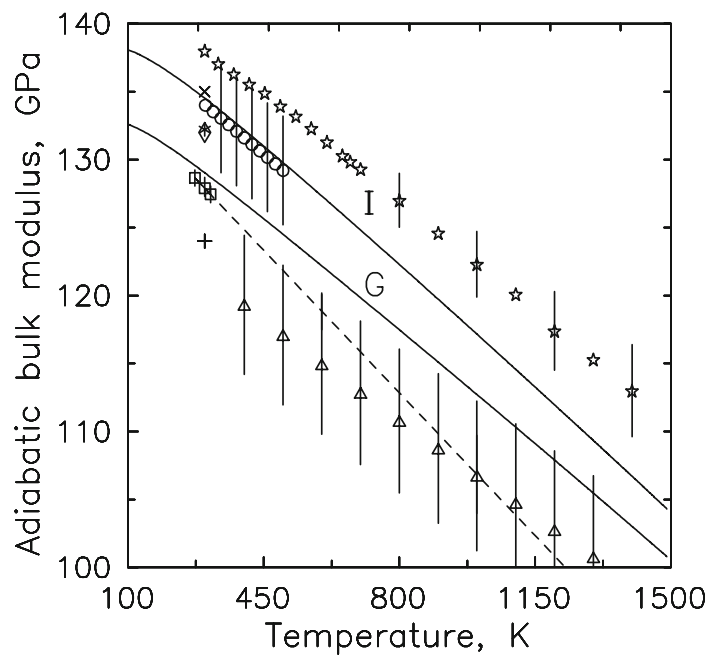

Fig. 2 Calculated adiabatic bulk modulus at 1 bar pressure using model $\mathrm{G}$ and model I. Experimental ultrasonic data are from Sumino (1979), star with points above $673 \mathrm{~K}$ extrapolated, Graham et al. (1988), square with extrapolated dashed line, Isaak et al. (1993), circle. Ultrasonic data at room conditions are from Akimoto (1972), diamond, Wang et al. (1989), asterisk. Results from static compression: Plymate and Stout (1990), triangle, Takahashi (1970), cross, Yagi et al. (1975), plus

Table 3 Electronic energy levels with their degeneracy in $\mathrm{Fe}_{2} \mathrm{SiO}_{4}$

\begin{tabular}{lllll}
\hline Assignment & $\begin{array}{l}\text { Energy/ } \\
\mathrm{cm}^{-1}\end{array}$ & Degeneracy & Site & References \\
\hline Ground state & 0 & 1 & M1 & Aronson et al. (2007) \\
Spin orbit & 27 & 1 & M1 & Aronson et al. (2007) \\
Spin orbit & 47 & 1 & M1 & Aronson et al. (2007) \\
Spin orbit & 92 & 2 & M1 & Aronson et al. (2007) \\
$\delta 1$ & 730 & 5 & M1 & Aronson et al. (2007) \\
$\delta 2$ & 1,500 & 5 & M1 & Aronson et al. (2007) \\
$e_{\mathrm{g}}$ & 8,060 & 5 & M1 & Burns (1985) \\
$e_{\mathrm{g}}$ & 11,060 & 5 & M1 & Burns (1985) \\
Ground state & 0 & 5 & M2 & Aronson et al. (2007) \\
$\delta_{1}$ & 1,670 & 5 & M2 & Burns (1985) \\
$\delta_{2}$ & 1,670 & 5 & M2 & Burns (1985) \\
$e_{g}$ & 8,830 & 5 & M2 & Burns (1985) \\
$e_{g}$ & 9,270 & 5 & M2 & Burns (1985) \\
\hline
\end{tabular}

function of temperature. In the second step we used these contributions together with the experimental heat capacity for temperatures below $300 \mathrm{~K}$ to derive the critical lambda contribution. The resulting heat capacity for the critical lambda effect was fitted with the one-parameter expression, resulting from Eq. 3. Figure 3 demonstrates that as a result of a different lattice vibrational contribution, the heat capacity and entropy for the critical lambda effect is slightly different from that established by Aronson et al. (2007). The heat capacity and entropy calculated with model $\mathrm{G}$ is not significantly different from that calculated with model I.

Figure 4 shows that sound wave velocities of Graham et al. (1988) and Isaak et al. (1993) are well represented by models $\mathrm{G}$ and I, respectively. Figure 4 also indicates that the transverse sound wave velocities given by Graham et al. (1988) are not significantly different from that reported by Isaak et al. (1993). The difference is significant for the longitudinal sound wave velocity. Because the adiabatic bulk modulus can be expressed in longitudinal and transverse sound wave velocity, the difference is also significant for the adiabatic bulk modulus as shown in Fig. 2.

Jacobs et al. (2001) were not able to discriminate between the thermal expansivity of Suzuki et al. (1981) and that measured by Smyth (1975) and Plymate and Stout (1990). Figure 5 shows that the vibrational analyses of model $G$ and I both prefer the thermal expansivity data measured by Suzuki et al. (1981) and are compatible with model A in the analysis of Jacobs et al. (2001). Table 4 indicates that our analyses prefer the ambient volume of Zhang et al. (1998), Richard and Richet (1990), Hazen (1977) and Smyth (1975). This value is lower than the recommended value by Jeanloz and Thompson (1983) used in the analysis by Jacobs et al. (2001). The $V-P-T$ data of Yagi et al. (1987), which are at the highest temperatures relative to all other measurements given in Table 2, are represented to within experimental uncertainty of about $0.3 \%$.

Figure 6 and Table 2 demonstrate that the heat capacity calculated from our analyses differs insignificantly from the experimental data for temperatures up to about $700 \mathrm{~K}$. For temperatures above $700 \mathrm{~K}$ the only experimental data are reported by Esser et al. (1933) on a natural fayalite sample and by Orr (1953) on a synthetic sample. The data of Orr (1953) are generally considered to be more accurate and are used in data compilations such as those by Barin (1989). From Eq. 36 and the data in Table 3, it can be readily shown that the electronic heat capacity contribution decreases for temperatures above $800 \mathrm{~K}$, when the spinorbit and $\delta$ energy levels become saturated. The contribution at $1,400 \mathrm{~K}$ is only about $4 \%$ of the total heat capacity. Figure 6 indicates that this contribution is not sufficient to explain the steep behavior of the heat capacity of Orr (1953) at high temperatures. We tried to explain this behavior by introducing intrinsic anharmonicity in our formalism, although no experimental data are available for it. Figure 6 indicates that this introduction leads to larger deviations from the data of Watanabe (1982) located in the temperature range between 350 and $700 \mathrm{~K}$ and that the temperature derivative of the heat capacity at temperatures larger than $1,000 \mathrm{~K}$ does not change significantly. In another session of calculations we tried volume dependent 


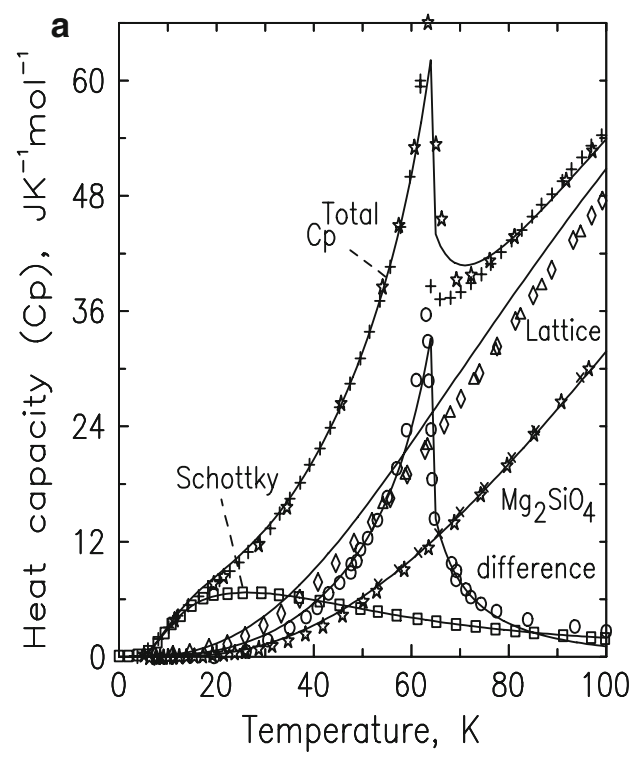

Fig. 3 Left panel calculated heat capacity at 1 bar pressure. Experimental data for the total heat capacity of fayalite are from Aronson et al. (2007), plus, Robie et al. (1982), star. Heat capacity data for forsterite are from Robie et al. (1982), star, Kelley (1943), cross. Heat capacity data for $\mathrm{Ca}_{2} \mathrm{SiO}_{4}$ are from King (1957), triangle. They are identical with those derived by Aronson et al. (2007), diamond. The Schottky contribution to the heat capacity derived by Aronson et al. (2007) is given by squares and that of the difference between the total heat capacity and the sum of the Schottky and lattice contribution is given by circles. The contributions denoted as 'Schottky', 'difference'

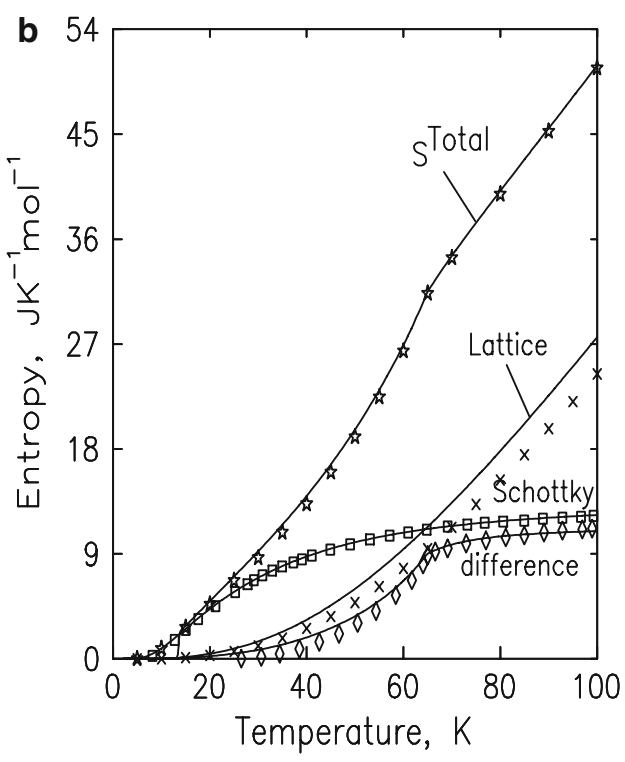

and 'lattice' are heat capacities at constant volume and appear as $C_{\mathrm{V}}^{\mathrm{el}-\mathrm{mg}}, C_{\mathrm{V}}^{\lambda}$ and $C_{\mathrm{V}}^{\mathrm{vib}}$ in Eq. 15 , respectively. Right panel calculated entropy corresponding to the heat capacity in the left panel. The total entropy is derived from the experimental $C_{\mathrm{P}}$ data from Robie et al. (1982), star. The crosses represent the entropy derived from the lattice heat capacity given by King (1957) and Aronson et al. (2007). Squares represent the Schottky contribution to entropy and diamonds the difference between the total entropy and the sum of the Schottky and lattice contribution
Fig. 4 Calculated longitudinal $\left(v_{\mathrm{L}}\right)$ and transverse $\left(v_{\mathrm{T}}\right)$ sound wave velocities at 1 bar pressure using model I and G. Experimental data are from Sumino (1979) with data above $600 \mathrm{~K}$ extrapolated using two methods, triangle, Graham et al. (1988), with extrapolated points above $T=350 \mathrm{~K}$, circle, and Isaak et al. (1993), square
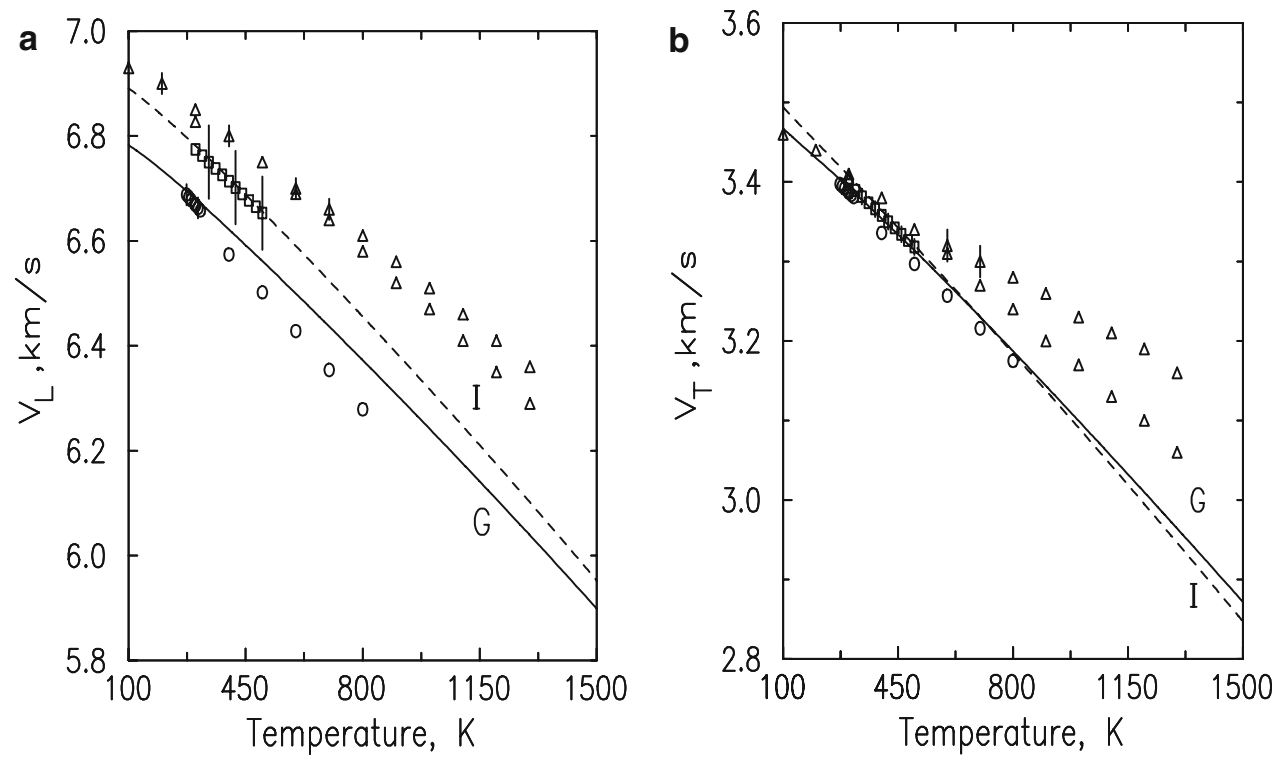

anharmonicity, $a_{j}=a_{j, 0}\left(V / V_{0}\right)^{\mathrm{m}}$, in which $a_{j, 0}$ represents the anharmonicity parameter of a specific mode $j$ at $0 \mathrm{~K}$. That results in the same unsuccessful effect on the heat capacity. Presently, we are not able to resolve the discrepancy between our calculations and the experimental data of Orr (1953). One explanation for this discrepancy, suggested by Hofmeister (1987), is that the electronic splittings are temperature dependent at high temperatures. Due to a lack of absorption spectral data at high temperatures, we did not consider in our calculations the temperature dependence of the electronic energy levels.

Models G and I produce a value for $K_{0}^{\prime}$ of 5.3 and 5.1, respectively, consistent with the value 5.2(4) measured by Graham et al. (1988) and Webb et al. (1984). The different 


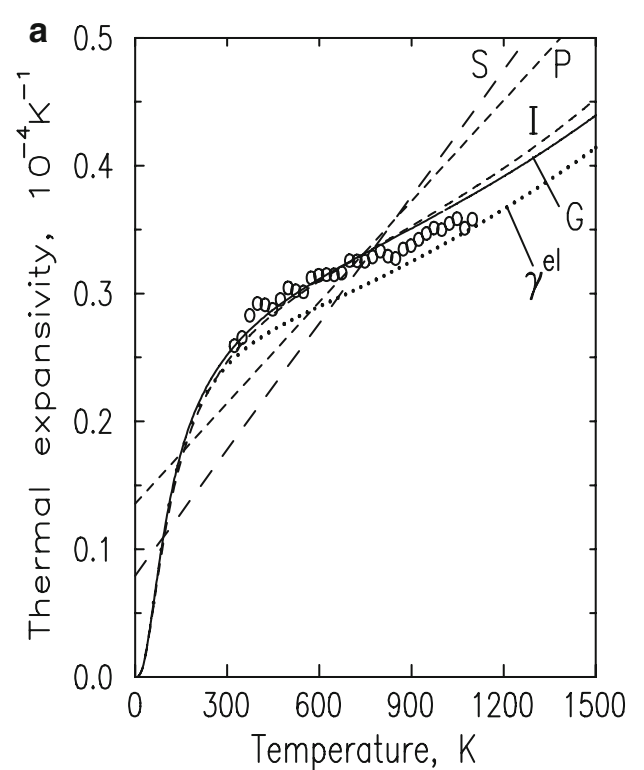

Fig. 5 a Calculated thermal expansivity at 1 bar pressure using model G (solid curve) and model I (dashed curve). Experimental data plotted as circles are from Suzuki et al. (1981). The dotted curve labeled with $\gamma^{\text {el }}$ is calculated using model $\mathrm{G}$ including electronic Grüneisen parameters given in "Effect of volume on electronic and magnetic properties of fayalite". The straight short dashed line is the thermal expansivity established by Plymate and Stout (1990) and the

Table 4 Ambient volume of $\mathrm{Fe}_{2} \mathrm{SiO}_{4}$, fayalite

\begin{tabular}{|c|c|c|}
\hline References & Method & $\begin{array}{l}\text { Volume } \\
\left(\mathrm{cm}^{3} / \mathrm{mol}\right)\end{array}$ \\
\hline Smyth (1975) & Single crystal X-ray & $46.222 \pm 0.012$ \\
\hline Yagi et al. (1975) & Powder diffraction & $46.350 \pm 0.102$ \\
\hline Hazen (1977) & Single crystal X-ray & $46.219 \pm 0.045$ \\
\hline Schwab and Küstner (1977) & Powder diffraction & $46.307 \pm 0.012$ \\
\hline Richard and Richet (1990) & Powder diffraction & $46.201 \pm 0.082$ \\
\hline Williams et al. (1990) & Powder diffraction & $46.341 \pm 0.066$ \\
\hline Zhang et al. (1998) & Single crystal X-ray & $46.206 \pm 0.009$ \\
\hline $\begin{array}{l}\text { Jeanloz and Thompson } \\
\text { (1983) }\end{array}$ & Recommended value & 46.27 \\
\hline Model G & $\begin{array}{l}\text { Calculated } \\
\qquad(T=293 \mathrm{~K})\end{array}$ & 46.236 \\
\hline Model I & $\begin{array}{l}\text { Calculated } \\
\qquad(T=293 \mathrm{~K})\end{array}$ & 46.217 \\
\hline
\end{tabular}

values calculated with model $\mathrm{G}$ and $\mathrm{I}$ indicate a trade-off between bulk modulus and its pressure derivative.

Effect of volume on electronic and magnetic properties of fayalite

Huggins (1974) investigated fayalite using Mössbauer Spectroscopy and came to the conclusion that the transition energy at $730 \mathrm{~cm}^{-1}$ for the M1 site decreases by approximately $7 \mathrm{~cm}^{-1} / \mathrm{GPa}$. Those at 1,500 and $1,670 \mathrm{~cm}^{-1}$ for

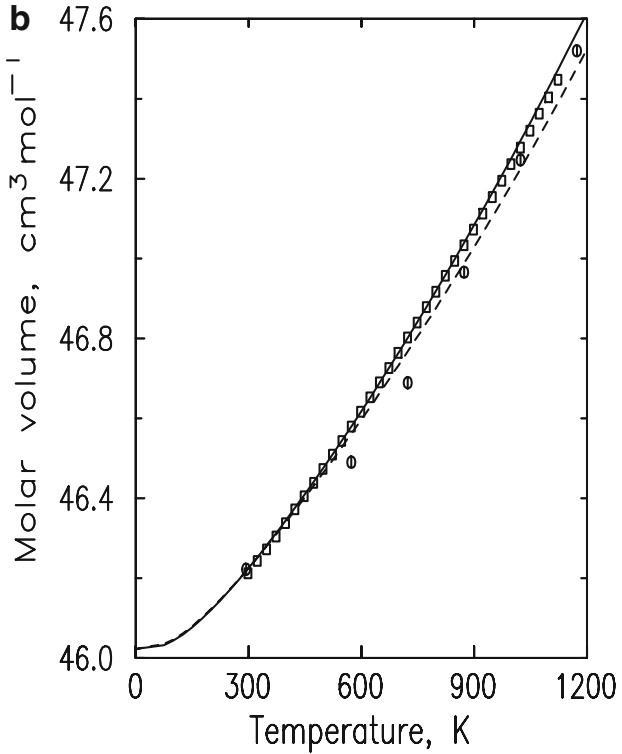

long dashed one that by Smyth (1975). b Calculated volume at 1 bar pressure using model I. The dashed curve has been calculated with model I and using electronic Grüneisen parameters given in "Effect of volume on electronic and magnetic properties of fayalite". Experimental data are from Smyth (1975), circle and Suzuki et al. (1981), square. Experimental uncertainties are comparable with the symbol size

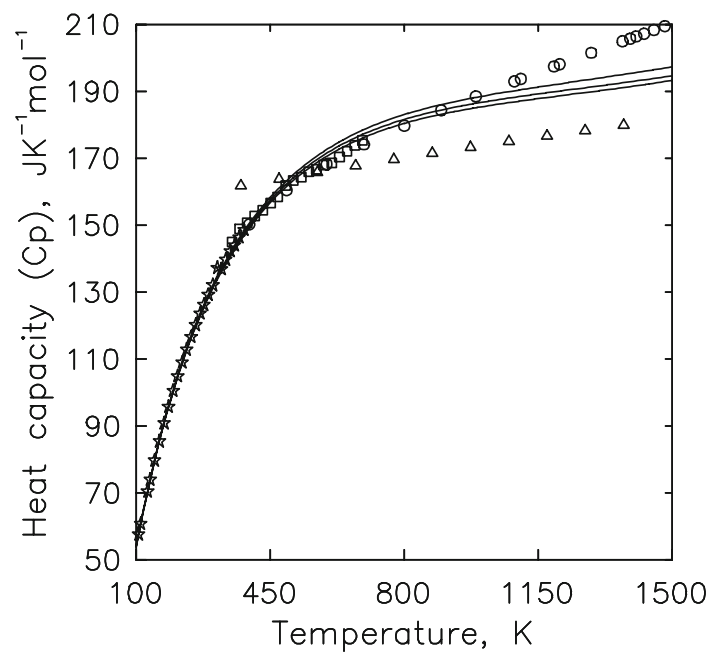

Fig. 6 Calculated heat capacity, $C_{\mathrm{P}}$, resulting from model $\mathrm{G}$ and $\mathrm{I}$ at 1 bar pressure for values of the anharmonicity parameters, $0,-10^{-5}$ and $-2 \times 10^{-5} \mathrm{~K}^{-1}$. ( $C_{\mathrm{P}}$ increases in this sequence). Experimental data are from Robie et al. (1982), star, Watanabe (1982), square, Orr (1953), circle and Esser et al. (1933), triangle

the M1 and M2 site, respectively, decrease by approximately $15 \mathrm{~cm}^{-1} / \mathrm{GPa}$. This decrease is associated with the decrease in distortion of M1 and M2 octahedra when pressure increases. Using the definition for the electronic Grüneisen parameter given by Eq. 31 it follows that a value for these parameters can be calculated using the relation 
$\gamma_{i}^{\mathrm{el}}=\frac{K}{\varepsilon_{i}}\left(\frac{\partial \varepsilon_{i}}{\partial P}\right)_{\mathrm{T}}$

with the ambient values 128 and $133 \mathrm{GPa}$ for the ambient isothermal bulk modulus for model $\mathrm{G}$ and I, respectively, we derive approximate values of $-1.25,-1.30$ and -1.17 for the electronic Grüneisen parameters associated with the $730,1,500$ and $1,670 \mathrm{~cm}^{-1}$ transition energies, respectively. Because in this case the contribution to the Helmholtz energy, $A^{\text {el-mg }}$, is volume dependent we investigated the additional effect of temperature through volume on the thermodynamic properties. At temperatures below room temperature the effect on thermodynamic properties is not significant compared with the experimental uncertainty in these properties. Although the effect on the electronic heat capacity can be as large as $7 \%$ at $1,500 \mathrm{~K}$, the effect on the total heat capacity is about $0.45 \%$ at this temperature, which is smaller than the experimental uncertainty of about $2 \%$. In addition to our findings in "Olivine solid solutions", the volume dependence of the energy transitions can also not explain the steep behavior of the heat capacity data of Orr (1953). However, Fig. 5 demonstrates that the effect of the electronic Grüneisen parameters on thermal expansivity and volume is quite significant, 7 and $0.28 \%$, respectively. The effect on sound wave velocities in the temperature range of the measurements of Graham et al. (1988) and Isaak et al. (1993) is about $0.04 \%$ for $V_{\mathrm{P}}$ and $0.27 \%$ for $V_{\mathrm{S}}$. At $1,500 \mathrm{~K}$ and 1 bar pressure it is $0.5 \%$ for $V_{\mathrm{P}}$ and $1.3 \%$ for $V_{\mathrm{S}}$. These $V_{\mathrm{P}}, V_{\mathrm{S}}$ values are not very different for conditions prevailing at $400 \mathrm{~km}$ depth in the Earth, 1,500 K and $13 \mathrm{GPa}$. The effect is 0.3 and $0.8 \%$ for $V_{\mathrm{P}}$ and $V_{\mathrm{S}}$, respectively.

The effect of volume on the lambda contribution to the Helmholtz energy, due to the antiferromagnetic-paramagnetic transition, given by Eq. 3 can be estimated by using the change of the Néel temperature with pressure as measured by Hayashi et al. (1987) using Mössbauer spectroscopy, $\left(\mathrm{d} T_{\mathrm{N}} / \mathrm{d} P\right)_{\mathrm{T}}=2.2 \pm 0.2 \mathrm{~K} / \mathrm{GPa}$. Differentiating Eq. 3 with respect to volume results in the pressure contribution

$P^{\lambda}=-n_{\mathrm{Fe}} R T \times$ const $\times\left(\frac{\partial g(\tau)}{\partial \tau}\right)_{\mathrm{T}} \frac{T K}{V T_{\mathrm{N}}^{2}}\left(\frac{\partial T_{\mathrm{N}}}{\partial P}\right)_{\mathrm{T}}$

Equation 17 shows for typical values, i.e., $130 \mathrm{GPa}$ for the isothermal bulk modulus and $46.2 \mathrm{~cm}^{3} / \mathrm{mol}$ for the volume at $300 \mathrm{~K}$, that at ambient conditions the contribution to pressure is about $21,700 \mathrm{~Pa}$, which is about $10^{-3} \%$ of the static and vibrational contributions to pressure. The effect of the change in Néel temperature with pressure on volume is small compared to the experimental uncertainty in volume, about $2 \times 10^{-5} \%$. The product $P^{\lambda} V$ is also small and therefore the assumption we have made in "Electronic and magnetic contributions to the Helmholtz energy in $\mathrm{Fe}_{2} \mathrm{SiO}_{4}$ " that the Helmholtz energy is approximately equal to the Gibbs energy is quite reasonable. A similar calculation at $100 \mathrm{~K}$ shows that the effect on the calculated volume is about $2 \times 10^{-3} \%$. The effect is larger at temperatures below the Néel temperature. For instance, at $30 \mathrm{~K}$ the effect on volume is about $0.04 \%$, which is comparable with the uncertainty in the ambient volume. Because the Néel temperature changes from $65 \mathrm{~K}$ to about $100 \mathrm{~K}$ in the pressure range between 0 and $16 \mathrm{GPa}$, the effect on magnetic entropy and magnetic heat capacity at temperatures between 0 and $100 \mathrm{~K}$ can be considerable at higher pressures and can even reach values of more than $100 \%$. However, at temperatures above $100 \mathrm{~K}$ this effect disappears with increasing temperature. Ignoring the change in Néel temperature with pressure leads to a difference in calculated total entropy of about $0.03 \%$ at $200 \mathrm{~K}$ and $3 \times 10^{-3} \%$ at $300 \mathrm{~K}$. For the total heat capacity the difference is 0.18 and $0.02 \%$ at 200 and $300 \mathrm{~K}$, respectively.

In summary, we conclude that our formalisms, which we used to calculate electronic, magnetic and lambda contributions to the Helmholtz energy, suggest that at mantlerelevant conditions the volume dependence of electronic energy transitions has a larger effect on thermodynamic properties than the change of the Néel temperature with pressure. At temperatures below room temperature the change in the Néel temperature with pressure is significant.

\section{Olivine solid solutions}

Our goal in this section is to calculate the vibrational and static lattice contribution to the thermodynamic mixing properties. Because magnetic and electronic contributions in our model depend only on the temperature, the volume derivative of the Helmholtz energy does not depend on these contributions. In our calculations we have neglected site preference for $\mathrm{M} 1$ and $\mathrm{M} 2$ of $\mathrm{Mg}^{2+}$ and $\mathrm{Fe}^{2+}$ ions, which could affect the results of our calculations. Dachs et al. (2007) give an overview of studies to the site preference of $\mathrm{Fe}^{2+}$ ions as a function of temperature, and indicate that a number of these studies produce incompatible results. To our knowledge a theoretical model which explains the temperature-composition behavior of the site preference has not been presented to date. Ignoring the site preference in olivine the volume is calculated from static lattice and vibrational contributions only, as is expressed by Eq. 11. Figure 7a shows an example of how the volume of a $\left(\mathrm{Mg}_{0.5}, \mathrm{Fe}_{0.5}\right)_{2} \mathrm{SiO}_{4}$ solid solution is determined from Eq. 11. The steep curve labeled with $P^{\text {st }}$ has been calculated with the first term on the right-hand side of Eq. 11 . The total pressure, denoted by $P^{\mathrm{st}}+P^{\mathrm{vib}}$ in Fig. 7a, matches the external pressure of 1 bar at a volume slightly larger than that for the ideal mixture indicated by the 
triangle. The difference in volume indicated by the circle and the triangle is the excess volume calculated using Eq. 12. Our choice of the excess contribution to the static lattice in the Helmholtz energy is rather arbitrary, but Fig. $7 \mathrm{~b}$ and Table 5 indicate that calculated excess volumes of olivine mixtures are not significantly different from the experimental data of Schwab and Küstner (1977) and Fisher and Medaris (1969). The data of Schwab and Küstner (1977) show the smallest experimental uncertainty and model $\mathrm{G}$ represents them more accurately than model I. This difference in accuracy is caused by the smaller bulk modulus of fayalite at ambient conditions based on model $\mathrm{G}$ relative to model I. Our model indicates that the bulk modulus of fayalite should be closer to that of forsterite than suggested by Isaak et al. (1993). Figure 7b shows that excess volume decreases with temperature and increases with pressure. According to models $\mathrm{G}$ and I the excess volume at Earth's mantle transition zone is not significantly different from the 1-bar experimental data of Schwab and Küstner (1977). The calculation of thermodynamic properties using our vibrational formalism is more complicated than that used by Jacobs and de Jong (2005b), in which we used polynomial functions to represent excess properties. For transparency we have calculated excess properties, including excess volume, in pressure-temperaturecomposition space with our vibrational formalism and

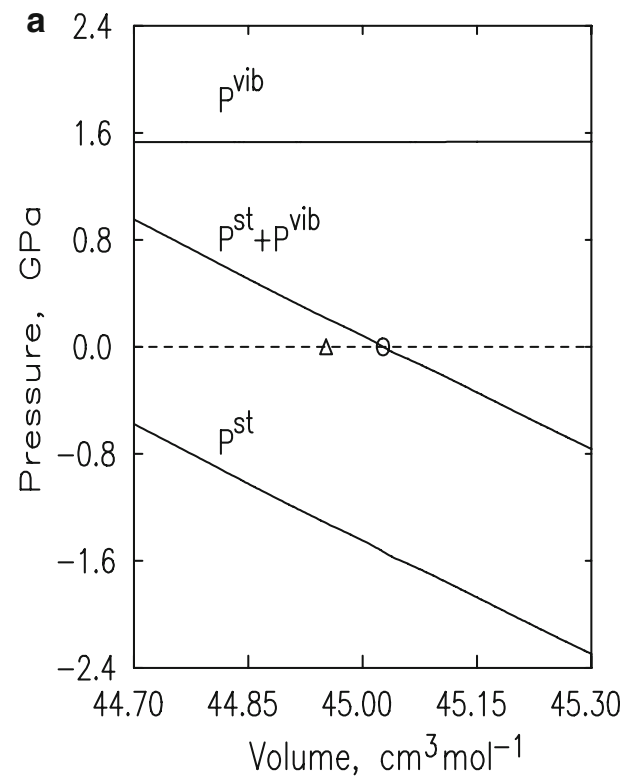

Fig. 7 a Calculated pressure contributions for $\left(\mathrm{Mg}_{0.5}, \mathrm{Fe}_{0.5}\right)_{2} \mathrm{SiO}_{4}$ at $300 \mathrm{~K}$ and an external pressure of 1 bar. $P^{\text {vib }}$ represents the vibrational and $P^{\text {st }}$ the static lattice contribution. The volume is calculated with Eq. 11 by $P^{\mathrm{st}}+P^{\mathrm{vib}}=10^{-4} \mathrm{GPa}$. The circle represents the resulting volume of the mixture and the triangle the volume of the ideal mixture, which is the average volume of $\mathrm{Mg}_{2} \mathrm{SiO}_{4}$ and $\mathrm{Fe}_{2} \mathrm{SiO}_{4}$ at this condition. The vibrational pressure contribution of the mixture changes by only $0.0024 \mathrm{GPa}$ in the indicated volume range. subsequently fitted the results using polynomial functions. The results of these fits are given in Table 6.

Figure 8 and Table 5 show that our formalism expressed by Eq. 58 results in a calculated excess enthalpy, which is not significantly different from experimental data of Wood and Kleppa (1981) and those of Kojitani and Akaogi (1994). Figure 8 indicates that the contributions of the static lattice and lattice vibrations to the excess enthalpy are quite substantial. We investigated these contributions in detail by performing calculations of the energy and pressure terms in Eqs. 59 and 60, neglecting the electronic and magnetic terms contained in Eq. 61 . Because the excess volume is small, the total excess enthalpy is mainly dominated by the total excess energy. A more detailed calculation using Eq. 48 reveals that the vibrational contribution to the excess energy is only $-3 \%$ at $50 \mathrm{~mol} \% \mathrm{Fe}_{2} \mathrm{SiO}_{4}$. Equation 59 reveals that at this composition the value of the excess energy contribution of the static lattice is $3 \%$ larger than that of the excess enthalpy. The large differences of the static lattice and vibrational contributions to excess enthalpy are therefore dominated by the $P V-\sum\left(y_{i} P_{i} V_{i}\right)$ terms in Eqs. 59 and 60. Figure 8 demonstrates that the excess enthalpy does not change significantly in the temperature and pressure range of Earth's lower mantle and transition zone. For $50 \mathrm{~mol} \% \mathrm{Fe}_{2} \mathrm{SiO}_{4}$ at $13 \mathrm{GPa}$ and $1,500 \mathrm{~K}$, it is about $42 \%$

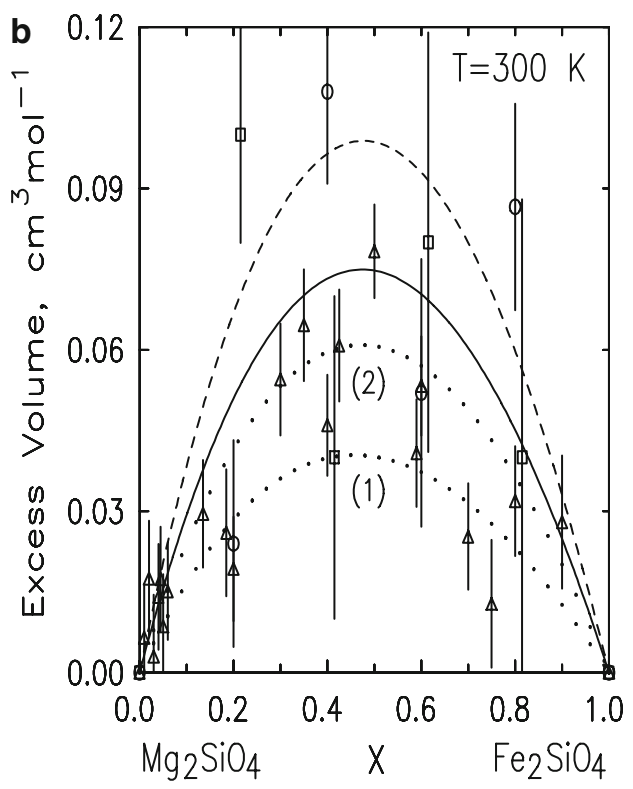

b Calculated excess volume at 1 bar pressure and $300 \mathrm{~K}$. The solid curve is calculated with model $\mathrm{G}$ and the dashed curve with model I. The dotted curve labeled (1) represents the excess volume calculated with model $\mathrm{G}$ at $1,473 \mathrm{~K}$ and 1 bar pressure and that labeled (2) at $1,473 \mathrm{~K}$ and $13 \mathrm{GPa}$. Experimental data are from Fisher and Medaris (1969), circles at 1 bar and squares at 2 bar and Schwab and Küstner (1977), triangles 
Table 5 Comparison of experimental thermodynamic properties and those predicted with model $\mathrm{G}$ and $\mathrm{I}$, respectively, for olivine ( $\mathrm{Mg}_{1-\mathrm{x}}$, $\left.\mathrm{Fe}_{\mathrm{x}}\right)_{2} \mathrm{SiO}_{4}$

\begin{tabular}{|c|c|c|c|c|c|}
\hline Property & $\begin{array}{l}\text { Maximum absolute } \\
\text { deviation in } \%, \mathrm{G}, \mathrm{I}\end{array}$ & $\begin{array}{l}\text { Average absolute } \\
\text { deviation in } \%, \mathrm{G}, \mathrm{I}\end{array}$ & $T$-range in $\mathrm{K}$ & $P$-range in GPa & References \\
\hline \multicolumn{6}{|l|}{ Fayalite } \\
\hline \multirow[t]{2}{*}{$V^{\mathrm{E}}$} & 112,178 & $48.2,67.5$ & 298 & 0.0 & Fisher and Medaris (1969) \\
\hline & 314,448 & $69.6,112$ & 298 & 0.0 & Schwab and Küstner (1977) \\
\hline \multirow[t]{2}{*}{$H^{\mathrm{E}}$} & 161,163 & $57.5,57.6$ & 975 & 0.0 & Wood and Kleppa (1981) \\
\hline & $15.1,14.5$ & $10.0,10.5$ & 975 & 0.0 & Kojitani and Akaogi (1990) \\
\hline$G^{\mathrm{E}}$ & 125,129 & $22.7,22.9$ & 1,473 & 0.0 & Nafziger and Muan (1967) \\
\hline $\mathrm{a}_{\mathrm{Mg}_{2} \mathrm{SiO}_{4}}$ & $9.44,9.44$ & $2.39,2.39$ & $1,423-1,473$ & 0.0 & Nafziger and Muan (1967) \\
\hline $\mathrm{a}_{\mathrm{Fe}_{2} \mathrm{SiO}_{4}}$ & $26.2,26.2$ & $7.53,7.52$ & $1,423-1,473$ & 0.0 & Nafziger and Muan (1967) \\
\hline $\mathrm{a}_{\mathrm{Fe}_{2} \mathrm{SiO}_{4}}$ & $18.7,18.6$ & $9.28,9.26$ & 1,477 & 0.0 & Kitayama and Katsura (1968) \\
\hline \multirow[t]{5}{*}{$K_{\mathrm{S}}$} & $4.60,4.70$ & $1.99,2.19$ & 298 & $0.0-13.0$ & Zaug et al. (1993) \\
\hline & $1.79,1.97$ & $0.91,1.05$ & 298 & $0.0-12.0$ & Abramson et al. (1997) \\
\hline & $8.94,8.94$ & $4.04,4.04$ & 298 & $0.0-32.3$ & Zha et al. (1998) \\
\hline & $3.88,4.22$ & $1.87,2.13$ & 298 & $0.0-7.1$ & Darling et al. (2004) \\
\hline & $0.46,0.24$ & $0.20,0.16$ & $300-1,200$ & 0.0 & Isaak (1992) \\
\hline \multirow[t]{5}{*}{$v_{\mathrm{B}}$} & $0.96,1.05$ & $0.49,0.58$ & 298 & $0.0-12.0$ & Abramson et al. (1997) \\
\hline & $4.53,4.55$ & $2.07,2.07$ & 298 & $0.0-32.3$ & Zha et al. (1998) \\
\hline & $0.81,0.98$ & $0.34,0.37$ & 298 & $0.0-7.1$ & Darling et al. (2004) \\
\hline & $0.22,0.13$ & $0.10,0.08$ & $300-1,200$ & 0.0 & Isaak (1992) \\
\hline & $0.97,1.04$ & $0.53,0.52$ & $300-1,600$ & 0.0 & Jackson et al. (2005) \\
\hline \multirow[t]{5}{*}{$v_{\mathrm{L}}$} & $1.51,1.56$ & $0.65,0.73$ & 298 & $0.0-12.0$ & Abramson et al. (1997) \\
\hline & $5.72,5.72$ & $2.37,2.38$ & 298 & $0.0-32.3$ & Zha et al. (1998) \\
\hline & $0.90,1.00$ & $0.32,0.36$ & 298 & $0.0-7.1$ & Darling et al. (2004) \\
\hline & $0.37,0.46$ & $0.28,0.37$ & $300-1,300$ & 0.0 & Isaak (1992) \\
\hline & $1.09,1.03$ & $0.32,0.36$ & $300-1,600$ & 0.0 & Jackson et al. (2005) \\
\hline \multirow[t]{5}{*}{$v_{\mathrm{T}}$} & $2.38,2.39$ & $0.92,0.93$ & 298 & $0.0-12.0$ & Abramson et al. (1997) \\
\hline & $8.33,8.36$ & $2.90,2.99$ & 298 & $0.0-32.3$ & Zha et al. (1998) \\
\hline & $1.02,1.04$ & $0.40,0.41$ & 298 & $0.0-7.1$ & Darling et al. (2004) \\
\hline & $0.89,0.88$ & $0.78,0.77$ & $300-1,300$ & 0.0 & Isaak (1992) \\
\hline & $1.41,1.39$ & $0.64,0.63$ & $300-1,600$ & 0.0 & Jackson et al. (2005) \\
\hline \multirow[t]{3}{*}{ Heat capacity } & $36.0,33.0$ & $4.10,3.77$ & $5-300$ & 0.0 & Dachs et al. (2007) \\
\hline & $2.01,1.85$ & $0.55,0.51$ & $80-300$ & 0.0 & Dachs et al. (2007) \\
\hline & $2.60,2.08$ & $0.79,0.79$ & $350-700$ & 0.0 & Watanabe (1987) \\
\hline \multirow[t]{3}{*}{ Volume } & $0.94,0.98$ & $0.23,0.25$ & 298 & $0.0-32.3$ & Zha et al. (1998) \\
\hline & $0.17,0.21$ & $0.07,0.10$ & 298 & $0.0-12.0$ & Abramson et al. (1997) \\
\hline & $0.31,0.29$ & $0.19,0.17$ & $298-1,073$ & $0.0-8.2$ & Liu and Li (2006) \\
\hline
\end{tabular}

larger relative to the value at $975 \mathrm{~K}$ and 1 bar, which is still within the uncertainty of the experiments.

Figure 9 demonstrates that the contributions of the static lattice and lattice vibrations to the excess Gibbs energy are also large. The static lattice contribution to the excess Gibbs energy is completely determined by the static lattice contribution to the excess enthalpy. The vibrational contribution to the excess Gibbs energy at $50 \mathrm{~mol} \% \mathrm{Fe}_{2} \mathrm{SiO}_{4}$ is for $87 \%$ determined by the vibrational contribution to excess enthalpy and for $13 \%$ by the excess entropy multiplied by the temperature. Nafziger and Muan (1967) carried out their experiments in a temperature range between 1,423 and $1,473 \mathrm{~K}$. This temperature range combined with their uncertainty of about 0.015 in the measured activities does not allow the determination of excess entropy from their experiments with sufficient accuracy. Therefore, Jacobs and de Jong (2005b) did not make an attempt to derive an excess contribution to entropy and based the excess Gibbs energy solely on the experimental enthalpy data of Wood and Kleppa (1981) and Kojitani and Akaogi (1994). The resulting excess Gibbs energy of Jacobs and de Jong 
Table 6 Parameterizations of excess properties of $\left(\mathrm{Mg}_{1}{ }_{y}, \mathrm{Fe}_{y}\right)_{2} \mathrm{SiO}_{4}$ solid solutions derived from results calculated with the vibrational formalism

\begin{tabular}{llllll}
\hline & $\mathrm{a}$ & $\mathrm{b}$ & $\mathrm{c}$ & $\mathrm{d}$ & $\mathrm{e}$ \\
\hline$V^{\mathrm{E}}$ & $0.1494(6)$ & $-5.7(5) \times 10^{-5}$ & 0 & $7.4(6) \times 10^{-4}$ & $1.4(7) \times 10^{-6}$ \\
$H^{\mathrm{E}}$ & $4,770(36)$ & $0.8(1)$ & $-7.3(5) \times 10^{-4}$ & $164.8(7)$ & 0 \\
$S^{\mathrm{E}}$ & $0.66(60)$ & 0 & 0 & $0.014(2)$ & 0 \\
\hline
\end{tabular}

The excess property $Z^{\mathrm{E}}$ is based on one-site mixing and is described with $Z^{\mathrm{E}}=Z(P, T) y(1-y)$, where $Z(P, T)=a+b \times(T-$ $300)+c \times(T-300)^{2}+d \times P+e \times(T-300) \times P$ with $T$ in Kelvin and $P$ in GPa. Excess volume is expressed in $\mathrm{cm}^{3} / \mathrm{mol}^{2}$ excess enthalpy in $\mathrm{J} / \mathrm{mol}$ and excess entropy in $\mathrm{J} / \mathrm{K} / \mathrm{mol}$

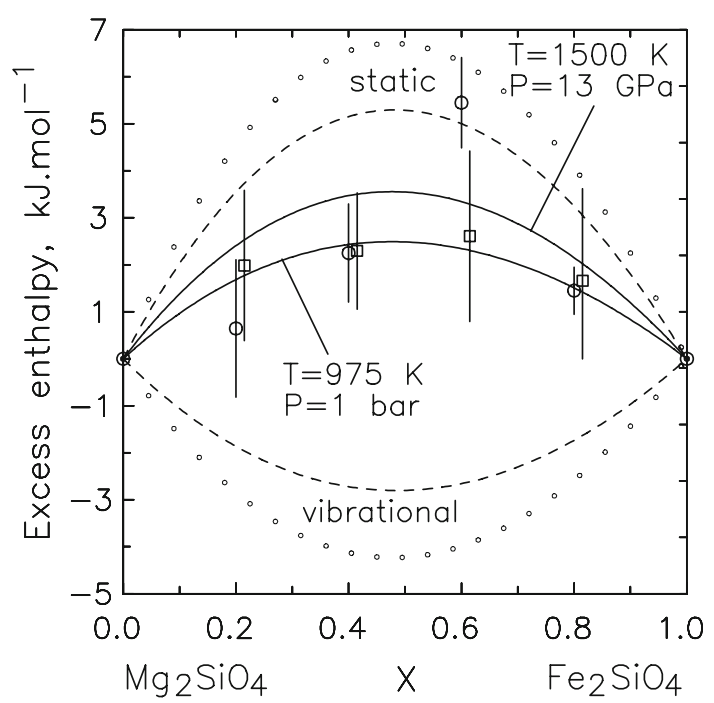

Fig. 8 Calculated excess enthalpy at 1 bar pressure, solid curves. The dashed curves represent the contribution to the excess enthalpy from the static lattice and lattice vibrations for model G, the dotted ones for model $\mathrm{I}$. The contribution of $P V^{\mathrm{E}}$ to the excess energy at 1 bar pressure is less than $5 \times 10^{-4} \%$. Experimental data are from Wood and Kleppa (1981), circle. For transparency the data Kojitani and Akaogi (1994), square, are offset by 0.015 in composition towards $\mathrm{Fe}_{2} \mathrm{SiO}_{4}$

(2005b) is at the edge of the uncertainty of the experiments whereas the present prediction represents the data of Nafziger and Muan (1967) to within experimental uncertainty.

In "Effect of volume on electronic and magnetic properties of fayalite", we showed that the implementation of electronic Grüneisen parameters in the description for fayalite affects its calculated volume and thermal expansivity. However, the effect on the excess properties is negligible compared to the uncertainties in the experimental data. These are about $1,0.27$, and $0.1 \%$ for excess Gibbs energy, excess volume, and excess enthalpy, respectively.

Because the excess Gibbs energy is positive the system $\mathrm{Mg}_{2} \mathrm{SiO}_{4}-\mathrm{Fe}_{2} \mathrm{SiO}_{4}$ has a miscibility gap. Figure $10 \mathrm{dem}$ onstrates that the effect of lattice vibrations on the location of the critical temperature of the predicted miscibility gap is substantial. Figure 9 indicates that the total excess Gibbs

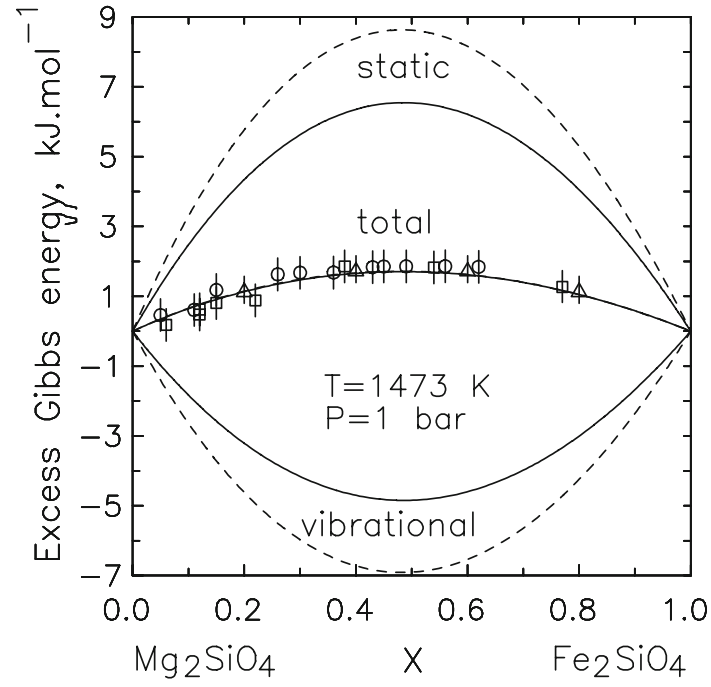

Fig. 9 Calculated contributions to the excess Gibbs energy at 1 bar pressure and $1,473 \mathrm{~K}$. The solid curves are calculated with model $\mathrm{G}$ and the dashed ones with model I. The total excess Gibbs energy calculated with model I is insignificantly different from that calculated with model G. Experimental data are from Nafziger and Muan (1967), circles obtained from olivine pyroxene equilibria, squares obtained from olivine and oxide equilibria, and Wiser and Wood (1991), triangles

energy is smaller than that of the excess Gibbs energy contribution of the static lattice. Therefore the critical temperature of the miscibility gap calculated using the total excess Gibbs energy is lower. Kojitani and Akaogi (1994) performed calorimetric measurements to establish the heat of mixing from which they derived a mixing parameter, $W_{\mathrm{H}, \mathrm{Mg}-\mathrm{Fe}}=5.3 \pm 1.7 \mathrm{~kJ} / \mathrm{mol}$, for a regular solution model based on single site mixing. They calculated from the available excess Gibbs energy data a value for the mixing parameter of the excess entropy: $W_{\mathrm{S}, \mathrm{Mg}-\mathrm{Fe}}=0.6 \pm$ $1.5 \mathrm{JK}^{-1} \mathrm{~mol}^{-1}$. Table 6 indicates that this value agrees well with our predicted value for this parameter. It also agrees with the value $-1.6 \pm 1.7 \mathrm{JK}^{-1} \mathrm{~mol}^{-1}$ established experimentally by Dachs et al. (2007). Table 6 gives a summary of excess properties represented by polynomial functions, obtained by fitting results predicted with our vibrational formalism. 


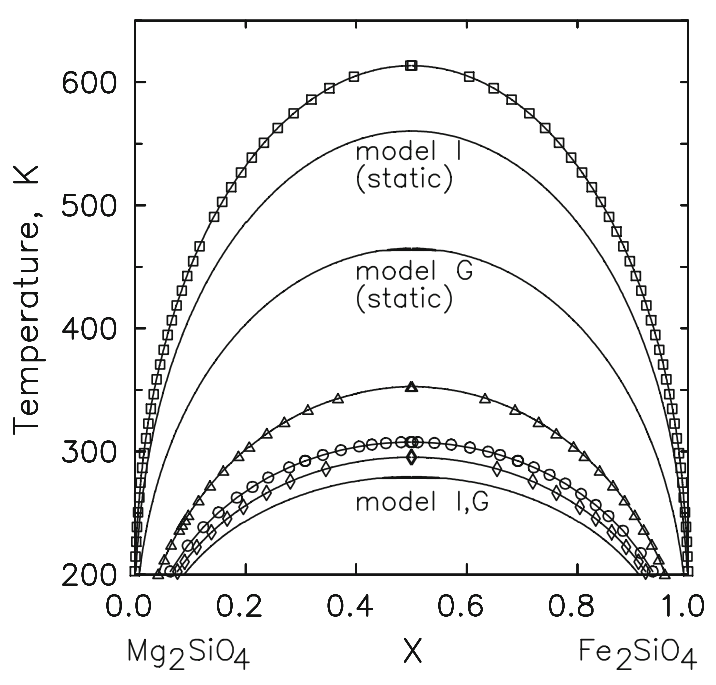

Fig. 10 Calculated miscibility gap in the system $\mathrm{Mg}_{2} \mathrm{SiO}_{4}-\mathrm{Fe}_{2} \mathrm{SiO}_{4}$ at 1 bar pressure. The calculations are based on one-site mixing basis using a regular solution model: Sack and Ghiorso (1989) squares, $W_{\mathrm{G}, \mathrm{Mg}-\mathrm{Fe}}=10.2 \pm 0.3 \mathrm{~kJ} / \mathrm{mol}$, Dachs et al. (2007), triangles, $W_{\mathrm{H}, \mathrm{Mg}-\mathrm{Fe}}=5.3 \pm 1.7 \mathrm{~kJ} / \mathrm{mol}$ and $W_{\mathrm{S}, \mathrm{Mg}-\mathrm{Fe}}=-1.6 \mathrm{~J} / \mathrm{K} / \mathrm{mol}$, Kojitani and Akaogi (1994), circles, $W_{\mathrm{H}, \mathrm{Mg}-\mathrm{Fe}}=5.3 \pm 1.7 \mathrm{~kJ} / \mathrm{mol}$ and $W_{\mathrm{S}, \mathrm{Mg}-\mathrm{Fe}}=0.6 \mathrm{~J} / \mathrm{K} / \mathrm{mol}$, Jacobs and de Jong (2005b), diamonds, $W_{\mathrm{G}, \mathrm{Mg}-\mathrm{Fe}}=4.9 \pm 0.5 \mathrm{~kJ} / \mathrm{mol}$. Our present prediction of the miscibility gap is labeled with model I and G. The curves labeled with static were calculated ignoring lattice vibrations

\section{Excess properties at low temperature}

We have assumed in our calculations using Eq. 10 that excess properties are determined by contributions due to the static lattice, due to lattice vibrations, and due to the antiferromagnetic-paramagnetic transition. As shown in Appendix 3, excess energy and entropy due to the antiferromagnetic-paramagnetic transition become significant for temperatures below $100 \mathrm{~K}$. Dachs and Geiger (2007) and Dachs et al. (2007) demonstrated by calorimetric measurements that at temperatures below $100 \mathrm{~K}$ excess contributions occur in the heat capacity and that the Néel temperature changes with composition. Figure 11 shows that the excess heat capacity calculated by our formalism is dominated by the antiferromagnetic-paramagnetic transition whereas lattice vibrations have a negligible effect. Figure 11 demonstrates that our model for the lambda transition captures the trends in the excess heat capacity, but that the finer details cannot be represented. The position of the downward and upward peaks in the heat capacity determined by the Néel temperature of fayalite and of the actual olivine composition are represented well, but our model for the lambda transition is not sophisticated enough to represent the shape and height of these peaks. Additionally, the experimental data at $30 \mathrm{~mol} \%$ fayalite indicate that the Schottky effect contributes to the excess heat capacity, whereas we have neglected its compositional

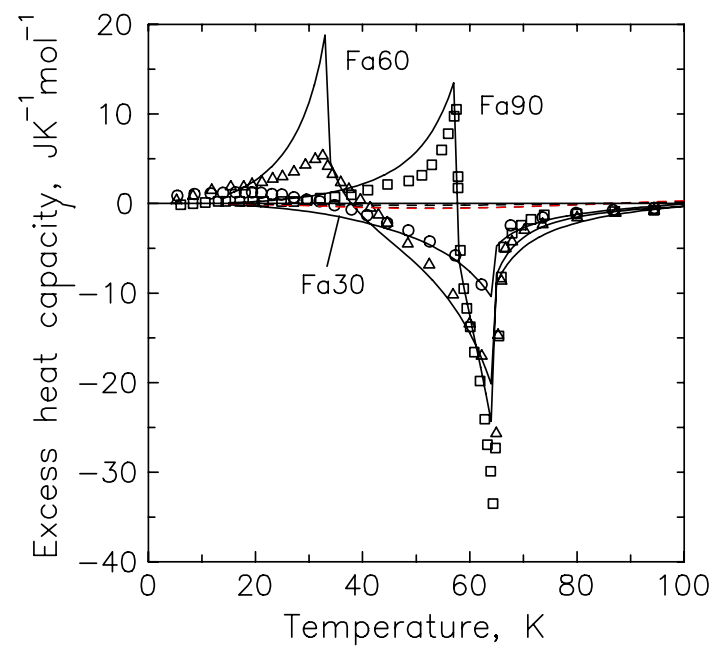

Fig. 11 Calculated excess heat capacity at 1 bar pressure. Experimental data are from Dachs and Geiger (2007) and Dachs et al. (2007). The difference between the result calculated with model G and $\mathrm{I}$ is about $1.7 \%$ and cannot be distinguished on the scale of the plot. The dashed curves close to $C_{P}^{\mathrm{E}}=0$ calculated with model I and $\mathrm{G}$ are the vibrational contributions to the excess heat capacity

variation. Improving the description of the excess heat capacity requires a more sophisticated model for the compositional dependence of the electronic properties combined with an improved model for the lambda transition.

Sound wave velocities of olivine

Figure 12 and Table 5 show for a $\left(\mathrm{Mg}_{0.896}, \mathrm{Fe}_{0.104}\right)_{2} \mathrm{SiO}_{4}$ mixture that our predicted longitudinal and bulk sound velocities represent within experimental uncertainty the experimental data on polycrystalline samples measured by Jackson et al. (2005) and those of Isaak (1992) on a singlecrystal sample. The average deviation of our calculated sound velocities from these two combined data sets is comparable with the deviation obtained when these sound velocities are fitted linearly. Figure 12 shows that our predicted transverse sound velocity deviates from the linear fit of the combined data sets of Jackson et al. (2005) and Isaak (1992). According to Jackson et al. (2005) systematic offsets in the measurements are especially apparent in the transverse sound velocity and result from minor heterogeneity or a variable degree of microcracking in the specimens. They preferred data obtained using a tapered buffer rod because it gave cleaner echo interference and traveltime-frequency patterns. We found that our predicted velocities agree with the data of Jackson et al. (2005) for the 3-mm sample for which a tapered buffer rod was applied, the maximum and average deviation being 0.38 and $0.16 \%$, respectively. Figure 13 and Table 5 indicate for a $\left(\mathrm{Mg}_{0.892} \mathrm{Fe}_{0.108}\right)_{2} \mathrm{SiO}_{4}$ mixture that the predicted 


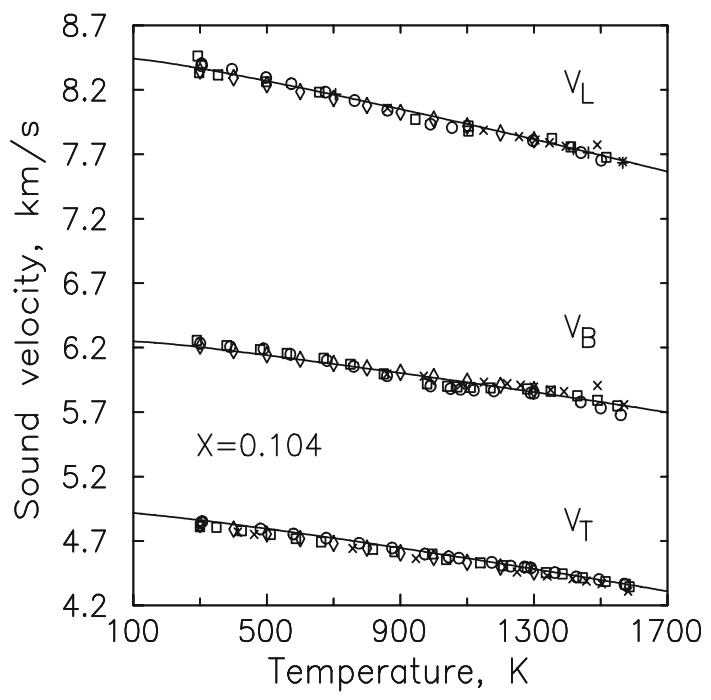

Fig. 12 Calculated sound wave velocities for $\left(\mathrm{Mg}_{0.896}, \mathrm{Fe}_{0.104}\right)_{2} \mathrm{SiO}_{4}$. Experimental data are from Isaak (1992) at 1 bar, diamond, and Jackson et al. (2005) at $300 \mathrm{MPa}$ : Circle $3 \mathrm{~mm}$ sample, tapered buffer rod, square $3 \mathrm{~mm}$ sample, untapered buffer rod, cross $5 \mathrm{~mm}$ sample, untapered buffer rod, cooling, plus $5 \mathrm{~mm}$ sample, untapered buffer rod, heating. Results of analyses $\mathrm{G}$ and I are not significantly different

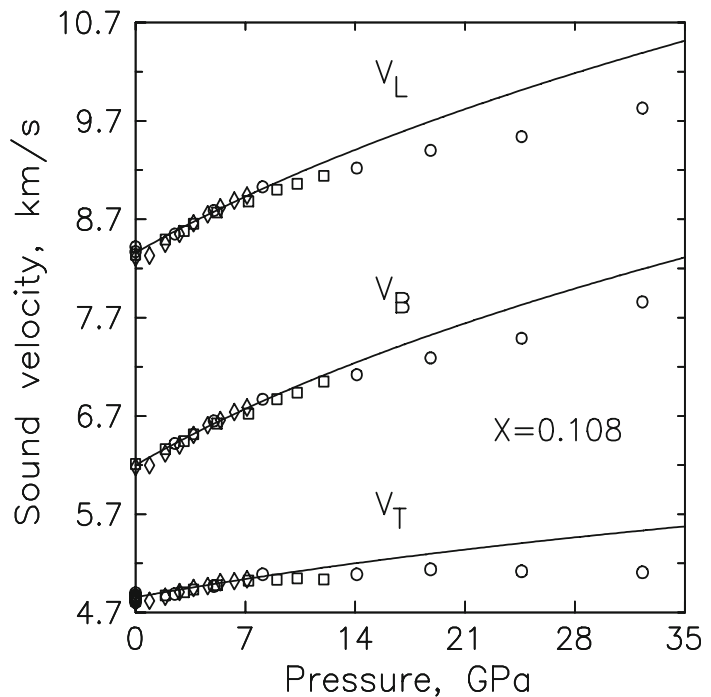

Fig. 13 Calculated sound wave velocities for $\left(\mathrm{Mg}_{0.892}, \mathrm{Fe}_{0.108}\right)_{2} \mathrm{SiO}_{4}$ at $300 \mathrm{~K}$. Experimental data are from: Abramson et al. (1997), square, Darling et al. (2004), diamond, Zha et al. (1998), circle. Results of analyses $\mathrm{G}$ and I are not significantly different

sound velocities agree well up to $10 \mathrm{GPa}$ with experimental data of Darling et al. (2004), Abramson et al. (1997) and Zha et al. (1998). Above $17 \mathrm{GPa}$, the last investigators found a non-linear behavior of shear elastic moduli with pressure, which may be associated with the onset of lattice instability when the material is compressed outside its stability field, resulting in amorphization of the material. This effect is not present in forsterite; see Jacobs and de Jong (2005a) and references therein. In our calculation we

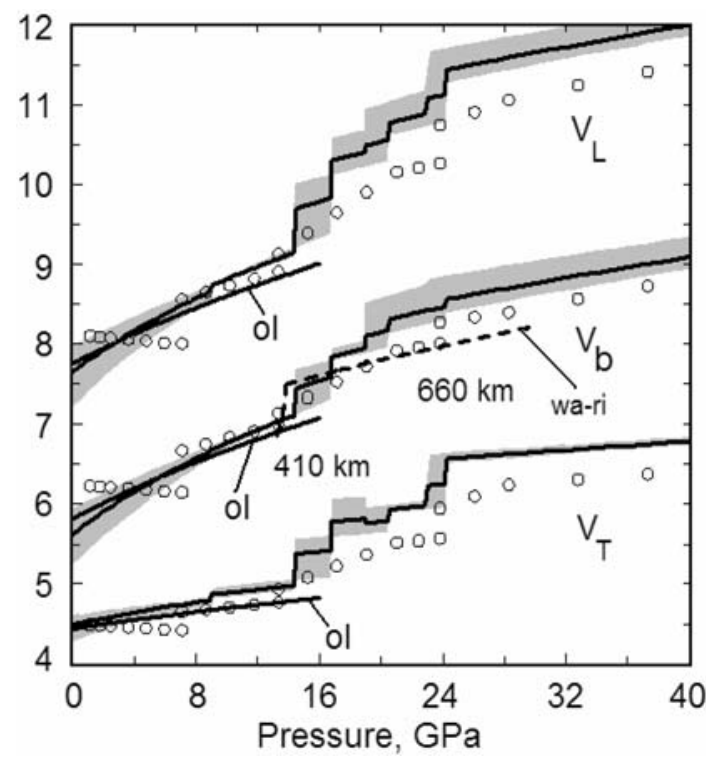

Fig. 14 Calculated longitudinal $\left(V_{\mathrm{L}}\right)$, bulk $\left(V_{\mathrm{B}}\right)$ and transverse $\left(V_{\mathrm{T}}\right)$ sound wave velocities. The gray fields represent sound velocities for compositions ranging from $\mathrm{Mg}_{2} \mathrm{SiO}_{4}$ to $\mathrm{MgSiO}_{3}$ along adiabats with a foot temperature of $1,420 \mathrm{~K}$. The solid curves are calculated for a mixture of $60 \mathrm{vol} \% \mathrm{Mg}_{2} \mathrm{SiO}_{4}$ and $40 \mathrm{vol} \% \mathrm{MgSiO}_{3}$. The solid curves labeled 'ol' are predicted using the present description for olivine $\left(\mathrm{Mg}_{0.9} \mathrm{Fe}_{0.1}\right)_{2} \mathrm{SiO}_{4}$ solid solution phase. The dashed curve labeled 'wa-ri' represents the bulk sound velocity for the wadsleyite and ringwoodite phases of $\left(\mathrm{Mg}_{0.9} \mathrm{Fe}_{0.1}\right)_{2} \mathrm{SiO}_{4}$ calculated with the description of Jacobs and de Jong (2005b). Circles represent sound velocities of PREM

did not attempt to include this effect for the olivine mixture, just as we did not in Jacobs and de Jong (2005b).

As we have indicated in Jacobs and de Jong (2007a) the application of our present model to realistic mantle compositions is exploratory because the effect of $\mathrm{Al}_{2} \mathrm{O}_{3}$ and $\mathrm{CaO}$ on phase equilibria and thermo physical properties has not been included. That also applies to the effect of grain size variation, which de Jong and Jacobs (2001) have shown to have a significant effect on sound wave velocities. In Jacobs and de Jong (2007) we presented predicted sound wave velocities and densities along isentropic paths for compositions between olivine and pyroxene in the system $\mathrm{MgO}-\mathrm{SiO}_{2}$. Figure 14 shows that sound wave velocities along an isentropic path with an adiabatic foot temperature of $1,420 \mathrm{~K}$, commensurate with a petrological study of Mercier and Carter (1975), are shifted to lower values relative to those calculated for mixtures of the magnesium endmembers of olivine and pyroxene in the system $\mathrm{MgO}-\mathrm{SiO}_{2}$. Figure 14 illustrates that at least iron should be incorporated in the all other phases of the $\mathrm{MgO}-$ $\mathrm{SiO}_{2}$ system. The calculated density along the adiabatic path for $\left(\mathrm{Mg}_{0.9} \mathrm{Fe}_{0.1}\right)_{2} \mathrm{SiO}_{4}$ resulting from our present description is not significantly different from that calculated using the description of Jacobs and de Jong (2005b). 


\section{Conclusions}

Because of the large difference in measured adiabatic bulk modulus for fayalite we carried out two thermodynamic analyses. One analysis is based on the sound velocity data of Graham et al. (1988), the other on the recommended sound velocity data of Isaak et al. (1993).

The difference in heat capacity, entropy and thermal expansivity for fayalite at 1 bar pressure calculated with either analysis is insignificant. Using new data of Aronson et al. (2007) we described the low temperature heat capacity and entropy, obtaining results in accordance with their experimental data and those of Robie et al. (1982) and Kelley (1943). Both analyses mimic heat capacity data of Watanabe (1982) between 350 and $700 \mathrm{~K}$. For temperatures above $700 \mathrm{~K}$ only two significantly different data sets are available, those of Esser et al. (1933) and those of Orr (1953). The set of Orr (1953) is generally considered to be the more accurate. However, our analyses do not agree with these data for temperatures above $1,000 \mathrm{~K}$. The difference between our calculated high temperature heat capacity and the data of Orr (1953) cannot be explained by including intrinsic anharmonicity, but may possibly be explained by temperature dependent electronic splittings. To check this requires additional electronic absorption spectra at temperatures above $1,000 \mathrm{~K}$.

Both analyses prefer the thermal expansivity of fayalite by Suzuki et al. (1981) and not those by Smyth (1975) and Plymate and Stout (1990). The use of electronic Grüneisen parameters to incorporate the pressure dependence of a number of electronic energy transitions has no significant effect on thermodynamic properties except for volume and thermal expansivity above room temperature. Their effect on sound wave velocities at a mantle condition of $1,500 \mathrm{~K}$ and $13 \mathrm{GPa}$ lies within the tomographic uncertainty of about $0.5 \%$. For a $\left(\mathrm{Mg}_{0.9} \mathrm{Fe}_{0.1}\right)_{2} \mathrm{SiO}_{4}$ olivine solid solution the effect on sound wave velocities is negligible.

The effect of pressure on the Néel temperature of fayalite significantly affects the heat capacity and entropy for temperatures below $100 \mathrm{~K}$. Above this temperature the effect is insignificant.

Our analyses prefer the ambient volume of fayalite by Zhang et al. (1998), Richard and Richet (1990), Hazen (1977) and Smyth (1975), which is lower than the recommended value given by Jeanloz and Thompson (1983). The difference between calculated volumes in pressuretemperature space produced by the two analyses is insignificant.

For olivine solid solutions we used a simple model for the static part of the Helmholtz by assuming that mixing takes place at the volume of the mixture. We combined this model with a phonon frequency-composition relation given by Lawson (1947) assuming one-mode behavior. Lack of experimental data compelled us to assume a linear composition dependence of the electronic and magnetic contributions to the Helmholtz energy. The combination of this model with either of the two thermodynamic analyses of fayalite predicts the excess volume, excess enthalpy and excess Gibbs energy to within experimental uncertainty. It indicates that within this model framework the excess electronic and magnetic effects are small for temperatures above room temperature in olivine solid solutions. Our formalism does well in representing trends in the excess heat capacity at temperatures below $100 \mathrm{~K}$, but not in representing the finer details of the experimental data. To improve the excess heat capacity description requires a more sophisticated model of the antiferromagnetic-paramagnetic lambda transition and of the composition dependence of electronic contribution to Helmholtz energy. Our model predicts that lattice vibrations have a negligible effect on excess heat capacity.

Although one-mode behavior produces satisfactory results for excess properties, additional Raman and infrared spectroscopic data are necessary to reveal the composition dependence of vibrational frequencies to resolve more details of two-mode behavior. This might necessitate a modification of the present thermodynamic description for olivine.

We found that lattice vibrations significantly affect the excess enthalpy and excess Gibbs energy but not the excess energy.

Our analysis based on the adiabatic bulk modulus data of Graham et al. (1988) predicts excess volume more accurately than the one based on the adiabatic bulk modulus data of Isaak et al. (1993).

We found that excess properties depend on pressure and temperature, but do not differ significantly at conditions prevailing at Earth's transition zone from those established by Jacobs and de Jong (2005b) using pressure and temperature independent polynomial parameterizations of excess entropy, enthalpy and volume.

Both analyses of fayalite result in predicted longitudinal and bulk sound velocities for olivine not significantly different from the measurements of Isaak (1992) and Jackson et al. (2005). Our predicted transverse sound velocity prefers the experimental data of Jackson et al. (2005) obtained for the 3-mm sample using a tapered buffer rod. Sound velocities at $300 \mathrm{~K}$ are consistent with experimental data up to about $17 \mathrm{GPa}$. Our calculations do not include the non-linear behavior of shear elastic moduli with pressure, which is indicative of lattice instability when olivine is compressed outside its stability field.

A comparison between our calculations and PREM shows that at least iron should be incorporated in the all other phases of the $\mathrm{MgO}-\mathrm{SiO}_{2}$ system. 
Acknowledgments This work was funded by the Netherlands Research Center for Integrated Solid Earth Sciences (ISES) for which we express our gratitude. We gratefully acknowledge constructive remarks and suggestions of two anonymous reviewers which improved the quality of the manuscript significantly.

Open Access This article is distributed under the terms of the Creative Commons Attribution Noncommercial License which permits any noncommercial use, distribution, and reproduction in any medium, provided the original author(s) and source are credited.

\section{Appendix 1: Helmholtz energy of pure endmembers}

The Helmholtz energy of an insulator material, such as $\mathrm{Mg}_{2} \mathrm{SiO}_{4}$ is given by

$$
A(T, V)=U^{\mathrm{ref}}\left(V_{0}^{\mathrm{st}}\right)+U^{\mathrm{st}}(V)+A^{\mathrm{vib}}(T, V) .
$$

The energy $U^{\text {ref }}\left(V_{0}^{\text {st }}\right)$ represents the energy contribution at zero Kelvin and zero pressure for a substance in which no vibrational motion of the atoms is present. It is adjusted such that the enthalpy of formation at $298.15 \mathrm{~K}$ and 1 bar pressure calculated from Eq. 18 represents the value reported in, e.g., the JANAF thermochemical tables compiled by Chase et al. (1985). The volume of this static crystal lattice is denoted by $V_{0}^{\text {st }}$ and the subscript ' 0 ' denotes the condition of zero pressure and zero Kelvin. The energy contribution $U^{\text {st }}(V)$ represents the change of the Helmholtz energy resulting from a change in volume of the static crystal lattice from $V_{0}^{\text {st }}$ to $V$. In the present work we prefer the equation of state derived by Vinet et al. (1987), to calculate this contribution

$$
\begin{aligned}
P^{\mathrm{st}}(V)= & 3 K_{0}^{\mathrm{st}}\left[\left(\frac{V}{V_{0}^{\mathrm{st}}}\right)^{-2 / 3}-\left(\frac{V}{V_{0}^{\mathrm{st}}}\right)^{-1 / 3}\right] \\
& \times \exp \left\{\frac{3}{2}\left(K_{0}^{\mathrm{st}}-1\right)\left[1-\left(\frac{V}{V_{0}^{\mathrm{st}}}\right)^{1 / 3}\right]\right\},
\end{aligned}
$$

$K_{0}^{\text {st }}$ represents the isothermal bulk modulus of the static lattice and $K_{0}^{\prime \text { st }}$ its pressure derivative. For $K_{0}^{\text {st }} \neq 1$ the energy $U^{\text {st }}(V)$ is given by integrating Eq. 19

$$
\begin{aligned}
U^{\mathrm{st}}(V)= & \frac{4 K_{0}^{\mathrm{st}} V_{0}^{\mathrm{st}}}{\left(K_{0}^{\prime \text { st }}-1\right)^{2}}\left\{1+\left[\frac{3}{2}\left(K_{0}^{\mathrm{st}}-1\right)\left(1-\left(\frac{V}{V_{0}^{\mathrm{st}}}\right)^{1 / 3}\right)-1\right]\right. \\
& \left.\times \exp \left[\frac{3}{2}\left(K_{0}^{\mathrm{st}}-1\right)\left(1-\left(\frac{V}{V_{0}^{\mathrm{st}}}\right)^{1 / 3}\right)\right]\right\}
\end{aligned}
$$

and for $K_{0}^{\text {st }}=1$ it is given by

$U^{\mathrm{st}}(V)=-9 K_{0}^{\mathrm{st}} V_{0}^{\mathrm{st}}\left[\left(\frac{V}{V_{0}^{\mathrm{st}}}\right)^{1 / 3}-\frac{1}{2}\left(\frac{V}{V_{0}^{\mathrm{st}}}\right)^{2 / 3}-\frac{1}{2}\right]$
The total pressure derived from Eq. 18 is

$P(T, V)=P^{\mathrm{st}}(V)+P^{\mathrm{vib}}(T, V)$.

In the present work the properties $V_{0}, K_{0}^{\text {st }}$ and $K_{0}^{\text {st }}$ and $U^{\text {ref }}\left(V_{0}^{\text {st }}\right)$ are fitting parameters obtained from a leastsquares optimization of available experimental data.

The vibrational contribution to the Helmholtz energy is calculated using Kieffer's (1979) model:

$$
\begin{aligned}
A^{\mathrm{vib}}(T, V)= & \frac{3 N_{\mathrm{A}}}{Z}\left(\frac{2}{\pi}\right)^{3} \sum_{i=1}^{3}\left[\int_{0}^{x_{i}} \frac{\arcsin ^{2}\left(x / x_{i}\right)}{\left(x_{i}^{2}-x^{2}\right)^{1 / 2}} A_{\mathrm{E}, i}^{\mathrm{vib}} \mathrm{d} x\right] \\
& +\left(3 n-\frac{3}{Z}\right) N_{\mathrm{A}} \sum_{j=1}^{N_{\mathrm{oc}}}\left[\int_{x_{l_{j}}}^{x_{u_{j}}} \frac{f_{j}}{x_{u_{j}}-x_{l_{j}}} A_{\mathrm{E}, j}^{\mathrm{vib}} \mathrm{d} x\right]
\end{aligned}
$$

where $A_{\mathrm{E}, i}^{\mathrm{vib}}$ represents the Helmholtz energy of an Einstein oscillator for mode $i, x=h v / k T$ with $h$ Planck's constant, $k$ Boltzmann's constant and $T$ temperature, $Z$ is the number of molecules in the primitive cell, $N_{\mathrm{A}}$ represents Avogadro's number and $n$ represents the number of atoms per molecule. The total degree of vibrational freedom for one mole of molecular formula unit is $3 n N_{\mathrm{A}}$ and $3 n Z$ represent the number of vibrational normal modes. The first term on the right-hand side of Eq. 22 represents the contributions due to acoustic lattice vibrations and the second term represents the optic modes of vibration. The total number of these modes is $N_{\text {OC }}$ and $f_{j}$ is the fraction of the total number of optic oscillators in mode $j$. These modes are represented by Einstein continua with lower and upper cut-off frequencies, respectively, determined by values for $x_{l_{j}}$ and $x_{u_{j}}$.

In the treatment of intrinsic anharmonicity we follow Wallace (1972) in that the substitution of the volume and temperature-dependent frequency into the quasi-harmonic expression for the entropy is to first order correct. The vibrational frequency of a particular mode is written as

$v(T, V)=v(V)(1+a T)$

Using Eq. 24 the Helmholtz energy of an Einstein oscillator for a particular mode is expressed as

$$
\begin{aligned}
A_{\mathrm{E}}^{\mathrm{vib}}(T, V)= & k T \sum_{j=0}^{m}\left[\frac{(a T)^{j}}{(1+a T)^{j+1}} \ln (1-\exp (-x))\right] \\
& +k T \sum_{j=1}^{m}\left[j \frac{(a T)^{j} x^{j+1}}{(1+a T)^{j+1}} \int_{\infty}^{x} \frac{\mathrm{d} x}{x^{j+1}(\exp (x)-1)}\right] \\
& +\frac{1}{2} k T \frac{x}{1+a T}
\end{aligned}
$$

The integral expressions in the second term on the righthand side were tabulated as function of $x$. We demonstrated in Jacobs and de Jong (2005a) that thermodynamic properties calculated from this power series expression, 
when $m \geq 4$, do not differ significantly from those calculated from Wallace's theorem, i.e., in which the Helmholtz energy is based on the numerical integration of the entropy.

The logarithm of the frequency of a vibrational mode $j$ is expanded in a Taylor series of $\ln (V)$ resulting in the expression

$\ln \left(\frac{v_{j}(V)}{v_{j, 0}}\right)=-\gamma_{j, 0} \ln \left(\frac{V}{V_{0}}\right)-\frac{1}{2} q_{1 j, 0} \cdot \gamma_{j, 0} \ln ^{2}\left(\frac{V}{V_{0}}\right)$

where $v_{j, 0}$ represents the cut-off vibrational frequency at zero pressure and zero temperature. The Grüneisen and mode $q$ parameters are defined by

$\gamma_{j, 0}=-\left(\frac{\partial \ln v_{j}}{\partial \ln V}\right)_{T=0, P=0}, \quad q_{1 j, 0}=\left(\frac{\partial \ln \gamma_{j}}{\partial \ln V}\right)_{T=0, P=0}$

The combination of Eqs. 24 and 26 expresses the frequency of a vibrational mode $j$ in temperature-volume space. The cut-off frequencies $v_{j, 0}$, mode Grüneisen parameters $\gamma_{j, 0}$, mode $q_{1 j, 0}$ parameters, and the mode anharmonicity parameters $a_{j, 0}$ are defined at zero Kelvin and zero pressure. These values are given in Table 1 for $\mathrm{Mg}_{2} \mathrm{SiO}_{4}$ and $\mathrm{Fe}_{2} \mathrm{SiO}_{4}$ and were derived from a leastsquares optimization of available experimental data.

In Kieffer's (1979) model the directionally averaged longitudinal and transverse sound velocities are related to the cut-off angular vibrational frequency $\omega_{i, \max }$ by

$u_{i}=\frac{1}{4}\left(\frac{4 \pi Z V}{3 N_{\mathrm{A}}}\right)^{1 / 3} \omega_{i, \max }$

where $u_{1}$ and $u_{2}$ represent the sound velocities of the transverse waves, $u_{3}$ that of the Voigt-Reuss-Hill longitudinal wave. The transverse sound velocity is calculated using the relation

$v_{T}^{2}=\frac{G}{\rho}=\frac{3}{4}\left(u_{3}^{2}-v_{\mathrm{B}}^{2}\right)=\frac{3}{4}\left(u_{3}^{2}-\frac{K_{\mathrm{S}}}{\rho}\right)$,

where $G$ is the shear modulus, $v_{\mathrm{B}}$ the bulk sound velocity, $K_{\mathrm{S}}$ the adiabatic bulk modulus and $\rho$ the density.

\section{Appendix 2: Calculation of electronic-magnetic contributions to thermodynamic properties}

This appendix gives a summary of the calculation of thermodynamic properties using the data given in Table 3 . To calculate thermodynamic properties relative to those at $0 \mathrm{~K}$ we write the partition function as

$Z=\frac{1}{g_{1}} \sum_{i=1}^{n} g_{i} \exp \left(-\frac{\varepsilon_{i}}{k T}\right)$
This expression results in zero contributions for the Helmholtz energy and entropy at $0 \mathrm{~K}$. To take volume dependence of the electronic energy levels into account we define an electronic Grüneisen parameter of energy level $i$ as

$\gamma_{i}^{\mathrm{el}}=-\left(\frac{\partial \ln \varepsilon_{i}}{\partial \ln V}\right)_{T}$

For fayalite not sufficient data are available to determine the volume dependence of the electronic Grüneisen parameters. The volume dependence of the energy $\varepsilon_{i}$ is given by

$\varepsilon_{i}(V)=\varepsilon_{i, 0}\left(\frac{V}{V_{0}}\right)^{\gamma_{i}^{\mathrm{el}}}$

The values for $\varepsilon_{i, 0}$ at ambient volume $V_{O}$ are given in Table 3. In the remaining part of this appendix $\varepsilon_{i} / k T$ is abbreviated to $x_{i}$. The expressions for the electronic contributions to thermodynamic properties are given below and are given per atom $\mathrm{Fe}$ and for one site, e.g., the M1 site.

Helmholtz energy

$A^{\mathrm{el}}=-k T \ln \left(\frac{1}{g_{1}} \sum_{i=1}^{n} g_{i} e^{-x_{i}}\right)$

Energy

$U^{\mathrm{el}}=k T \frac{\sum_{i=1}^{n} g_{i} x_{i} e^{-x_{i}}}{\sum_{i=1}^{n} g_{i} e^{-x_{i}}}$

Entropy

$S^{\mathrm{el}}=k\left\{\ln \left(\frac{1}{g_{1}} \sum_{i=1}^{n} g_{i} e^{-x_{i}}\right)+\frac{\sum_{i=1}^{n} g_{i} x_{i} e^{-x_{i}}}{\sum_{i=1}^{n} g_{i} e^{-x_{i}}}\right\}$

Heat capacity at constant volume

$C_{V}^{\mathrm{el}}=k\left\{\frac{\sum_{i=1}^{n} g_{i} x_{i}^{2} e^{-x_{i}}}{\sum_{i=1}^{n} g_{i} e^{-x_{i}}}-\left(\frac{\sum_{i=1}^{n} g_{i} x_{i} e^{-x_{i}}}{\sum_{i=1}^{n} g_{i} e^{-x_{i}}}\right)^{2}\right\}$

Contribution to pressure

$P^{\mathrm{el}}=\frac{k T}{V} \frac{\sum_{i=1}^{n} g_{i} \gamma_{i}^{\mathrm{el}} x_{i} e^{-x_{i}}}{\sum_{i=1}^{n} g_{i} e^{-x_{i}}}$

Contribution to temperature derivative of the pressure

$$
\begin{aligned}
\left(\frac{\partial P^{\mathrm{el}}}{\partial T}\right)_{V}= & \frac{k}{V\left(\sum_{i=1}^{n} g_{i} e^{-x_{i}}\right)^{2}}\left\{\sum_{i=1}^{n} g_{i} e^{-x_{i}} \sum_{i=1}^{n} g_{i} \gamma_{i}^{\mathrm{el}} x_{i}^{2} e^{-x_{i}}\right. \\
& \left.-\sum_{i=1}^{2} g_{i} \gamma_{i}^{\mathrm{el}} x_{i} e^{-x_{i}} \sum_{i=1}^{n} g_{i} x_{i} e^{-x_{i}}\right\}
\end{aligned}
$$


Contribution to isothermal bulk modulus

$$
\begin{aligned}
K^{\mathrm{el}}= & \frac{k T}{V \sum_{i=1}^{n} g_{i} e^{-x_{i}}} \\
& \times\left\{\sum_{i=1}^{n} g_{i} \gamma_{i}^{\mathrm{el}} x_{i}\left(1+\gamma_{i}^{\mathrm{el}}-\gamma_{i}^{\mathrm{el}} x_{i}\right)+\frac{\sum_{i=1}^{n} g_{i} \gamma_{i}^{\mathrm{el}} x_{i} e^{-x_{i}}}{\sum_{i=1}^{n} g_{i} e^{-x_{i}}}\right\}
\end{aligned}
$$

Contribution to Gibbs energy

$G^{\mathrm{el}}=A^{\mathrm{el}}+P^{\mathrm{el}} V$

\section{Appendix 3: Excess properties due to the lambda transition}

We adopt a method which is frequently used in the SGTE community by writing Eq. 3 as

$A^{\lambda}(T, \vec{y})=n_{\mathrm{Fe}} R T[g(\tau(\vec{y}))-1] \cdot c(\vec{y})$

From Eq. 41 it is derived that at high temperature the energy and entropy for the antiferromagneticparamagnetic transition reach constant values. In the case of fayalite constant values are reached for temperatures higher than $100 \mathrm{~K}$ considering the experimental error in entropy and energy values, $0.1 \mathrm{JK}^{-1} \mathrm{~mol}^{-1}$ and $600 \mathrm{Jmol}^{-1}$, respectively. As can be deduced from Eq. 4b the value of $g(\tau)$ is small at higher temperatures, typically $-2 \times 10^{-5}$ at $300 \mathrm{~K}$. Therefore we approximate Eq. 41 as

$A^{\lambda}(T, \vec{y})=-n_{\mathrm{Fe}} R T \cdot c(\vec{y})$

From Eq. 42 it follows that $U^{\lambda}(T, \vec{y})$ is zero and therefore no excess energy is present. We impose that at high temperature no excess contributions is present for the entropy as well by writing that

$A^{\lambda}(T, \vec{y})=y_{2} A_{2}^{\lambda}(T)$

Inserting Eq. 42 into Eq. 43 gives the composition dependence of $c(\vec{y})$ as:

$c(\vec{y})=-\frac{A_{2}^{\lambda}(T)}{n_{\mathrm{Fe}} R T} y_{2}=$ const $\cdot y_{2}$

For fayalite 'const' has the value given in Table 1. Equation 41 becomes:

$A^{\lambda}(T, \vec{y})=n_{\mathrm{Fe}} R T[g(\tau(\vec{y}))-1] \cdot$ const $\cdot y_{2}$

In our calculation of the excess properties at temperatures below room temperature, due to the antiferromagnetic-paramagnetic transition, we assume that Eq. 44 remains valid and that the non-linearity of the Helmholtz energy is due to larger values of $g(\tau(\vec{y}))$.

Dachs et al. (2007b) showed that the Néel temperature is linear with composition between 60 and $100 \mathrm{~mol} \%$ fayalite. At compositions less than $60 \mathrm{~mol} \%$ fayalite no transition between the antiferromagnetic and paramagnetic state could be established and thus no Néel temperature is present for these compositions. Therefore, we take the Néel temperature zero for compositions between 0 and $60 \mathrm{~mol} \%$ fayalite. Between 60 and $100 \mathrm{~mol} \%$ fayalite we fitted the Néel temperatures with the equation

$T_{\mathrm{N}}(\vec{y})=-13.66+78.54 \cdot y_{2}$

Because at high temperatures the value of $g(\tau)$ is small, Eq. 45 results in no excess contribution. When Eq. 45 is used for temperatures below about $100 \mathrm{~K}$, the magnetic Helmholtz energy is linear for compositions between 0 and $60 \mathrm{~mol} \%$ fayalite, because $\tau$ is infinitely large and $g(\tau)=0$. For compositions between 60 and $100 \mathrm{~mol} \%$ fayalite $\tau$ decreases and $g(\tau)$ attains values deviating from zero resulting in a non-linear Helmholtz energy. That results in an excess contribution to the Helmholtz energy due to the antiferromagnetic-paramagnetic transition, $A^{\mathrm{E} \lambda}(T, \vec{y})=A^{\lambda}(T, \vec{y})-y_{2} A_{2}^{\lambda}(T)$.

\section{Appendix 4: Contributions to excess properties}

The excess Helmholtz energy at selected $(T, P)$ is defined as

$A^{\mathrm{E}}(T, P, \vec{y})=A(T, P, \vec{y})-\sum_{i=1}^{2} y_{i} A_{i}(T, P)$

At the condition $(T, P)$ the volume of the mixture is $V$ and that for endmember $i$ is $V_{i}$ resulting in

$A^{\mathrm{E}}(T, V, \vec{y})=A(T, V, \vec{y})-\sum_{i=1}^{2} y_{i} A_{i}\left(T, V_{i}\right)$

Assuming that contributions of static lattice, lattice vibrations, electronic and magnetic to the Helmholtz energy are additive, the excess Helmholtz energy is partitioned as

$$
\begin{aligned}
A^{\mathrm{Est}}(V, \vec{y}) & =A^{\mathrm{st}}(V)-\sum_{i=1}^{2} y_{i} A_{i}^{\mathrm{st}}\left(V_{i}\right) \\
& =U^{\mathrm{st}}(V)-\sum_{i=1}^{2} y_{i} U_{i}^{\mathrm{st}}\left(V_{i}\right)=U^{\mathrm{Est}}(V, \vec{y})
\end{aligned}
$$

$A^{\mathrm{Evib}}(T, V, \vec{y})=A^{\mathrm{vib}}(T, V, \vec{y})-\sum_{i=1}^{2} y_{i} A_{i}^{\mathrm{vib}}\left(T, V_{i}\right)$

$A^{\text {Eel-mg }}(T, V, \vec{y})=A^{\mathrm{el}-\mathrm{mg}}(T, V, \vec{y})-\sum_{i=1}^{2} y_{i} A_{i}^{\mathrm{el}-\mathrm{mg}}\left(T, V_{i}\right)$

$A^{\mathrm{E} \lambda}(T, \vec{y})=A^{\lambda}(T, \vec{y})-\sum_{i=1}^{2} y_{i} A_{i}^{\lambda}(T)$

In Eq. 9 we have introduced the assumption that the Helmholtz energy of the mixture is given by 
$A^{\mathrm{st}}(V)=\sum_{i=1}^{2} y_{i} A_{i}^{\mathrm{st}}(V)=\sum_{i=1}^{2} y_{i} U_{i}^{\mathrm{st}}(V)$

The calculation of the excess energy and excess entropy proceeds in the same way with the difference that the excess entropy of the static lattice is zero.

The calculation of the excess enthalpy proceeds as follows. Starting from an analogous expression given by Eqs. 47 and 48 it follows that

$H^{\mathrm{E}}(T, P, \vec{y})=H^{\mathrm{E}}(T, V, \vec{y})=H(T, V, \vec{y})-\sum_{i=1}^{2} y_{i} H_{i}\left(T, V_{i}\right)$

Because enthalpy is derived from the energy this expression is written as

$H^{\mathrm{E}}(T, P, \vec{y})=U(T, V, \vec{y})+P V-\sum_{i=1}^{2} y_{i}\left\{U_{i}\left(T, V_{i}\right)+P V_{i}\right\}$

Because contributions of static lattice, lattice vibrations, electronic, magnetic and lambda transition effects to Helmholtz energy are assumed to be additive, this is also the case for energy and pressure. The pressure $P$, on the mixture is evaluated at the same value as for the endmembers. For the mixtures it is expressed as

$$
\begin{aligned}
P= & P(T, V, \vec{y}) \\
= & P^{\mathrm{st}}(V, \vec{y})+P^{\mathrm{vib}}(T, V, \vec{y})+P^{\mathrm{e} l-\mathrm{mg}}(T, V, \vec{y}) \\
& +P^{\lambda}(T, V, \vec{y})
\end{aligned}
$$

The same pressure on the pure endmembers is given by:

$$
\begin{aligned}
P & =P_{i}\left(T, V_{i}\right) \\
& =P_{i}^{\mathrm{st}}\left(V_{i}\right)+P_{i}^{\mathrm{vib}}\left(T, V_{i}\right)+P^{\mathrm{el}-\mathrm{mg}}\left(T, V_{i}\right)+P^{\lambda}\left(T, V_{i}\right)
\end{aligned}
$$

By combining Eqs. 55, 56 and 57 the excess enthalpy is written as:

$$
\begin{aligned}
H^{\mathrm{E}}(T, P, \vec{y})= & H^{\mathrm{Est}}(T, V, \vec{y})+H^{\mathrm{Evib}}(T, V, \vec{y}) \\
& +H^{\mathrm{Eel}-\mathrm{mg}}(T, V, \vec{y})+H^{\mathrm{E} \lambda}(T, V, \vec{y})
\end{aligned}
$$

where,

$$
\begin{aligned}
H^{\mathrm{st}}(T, P, \vec{y})= & U^{\mathrm{st}}(V, \vec{y})+P^{\mathrm{st}}(V, \vec{y}) \cdot V \\
& -\sum_{i=1}^{2} y_{i}\left\{U_{i}^{\mathrm{st}}\left(V_{i}\right)+P_{i}^{\mathrm{st}}\left(V_{i}\right) \cdot V_{i}\right\} \\
= & U^{\mathrm{Est}}(V, \vec{y})+P^{\mathrm{st}}(V, \vec{y}) \cdot V \\
& -\sum_{i=1}^{2} y_{i} \cdot P_{i}^{\mathrm{st}}\left(V_{i}\right) \cdot V_{i}
\end{aligned}
$$

$$
\begin{aligned}
H^{\mathrm{Evib}}(T, P, \vec{y})= & U^{\mathrm{vib}}(T, V, \vec{y})+P^{\mathrm{vib}}(T, V, \vec{y}) \cdot V \\
& -\sum_{i=1}^{2} y_{i}\left\{U_{i}^{\mathrm{vib}}\left(T, V_{i}\right)+P_{i}^{\mathrm{vib}}\left(T, V_{i}\right) \cdot V_{i}\right\} \\
= & U^{\mathrm{Evib}}(T, V, \vec{y})+P^{\mathrm{vib}}(T, V, \vec{y}) \cdot V \\
& -\sum_{i=1}^{2} y_{i} \cdot P_{i}^{\mathrm{vib}}\left(T, V_{i}\right) \cdot V_{i} \\
H^{\mathrm{Eel}-\mathrm{mg}}(T, P, \vec{y})= & U^{\mathrm{el}-\mathrm{mg}}(T, V, \vec{y})+P^{\mathrm{el}-\mathrm{mg}}(T, V, \vec{y}) \cdot V \\
& -\sum_{i=1}^{2} y_{i}\left\{U_{i}^{\mathrm{el}-\mathrm{mg}}\left(T, V_{i}\right)+P_{i}^{\mathrm{el}-\mathrm{mg}}\left(T, V_{i}\right) \cdot V_{i}\right\} \\
= & U^{\mathrm{Eel}-\mathrm{mg}}(T, V, \vec{y})+P^{\mathrm{el}-\mathrm{mg}}(T, V, \vec{y}) \cdot V \\
& -\sum_{i=1}^{2} y_{i} \cdot P_{i}^{\mathrm{el}-\mathrm{mg}}\left(T, V_{i}\right) \cdot V_{i} \\
H^{\mathrm{E} \lambda}(T, P, \vec{y})= & U^{\lambda}(T, V, \vec{y})+P^{\lambda}(T, V, \vec{y}) \cdot V \\
& -\sum_{i}^{\mathrm{E} \lambda} y_{i}\left\{U_{i}^{\lambda}\left(T, V_{i}\right)+P_{i}^{\lambda}\left(T, V_{i}\right) \cdot V_{i}\right\} \\
= & -\sum_{i=1}^{2} y_{i} \cdot P_{i}^{\lambda}\left(T, V_{i}\right) \cdot V_{i}
\end{aligned}
$$

The excess Gibbs energy contributions are calculated from the expressions for the excess entropy and excess enthalpy.

\section{References}

Abramson EH, Brown JM, Slutsky LJ, Zaug J (1997) The elastic constants of San Carlos olivine to $17 \mathrm{GPa}$. J Geophys Res 102:12253-12263. doi:10.1029/97JB00682

Akimoto S (1972) The system $\mathrm{MgO}-\mathrm{FeO}-\mathrm{SiO}_{2}$ at high pressures an temperatures-phase equilibria and elastic properties. Tectonophysics 13:161-187. doi:10.1016/0040-1951(72)90019-4

Andrault D, Bouhifd MA, Itié JP, Richet P (1995) Compression and amorphization of $(\mathrm{Mg}, \mathrm{Fe})_{2} \mathrm{SiO}_{4}$ olivines: an X-ray diffraction study up to $70 \mathrm{GPa}$. Phys Chem Miner 22:99-107. doi:10.1007/ BF00202469

Aronson MC, Stixrude L, Davis MK, Gannon W, Ahilan K (2007) Magnetic excitations and heat capacity of fayalite, $\mathrm{Fe}_{2} \mathrm{SiO}_{4}$. Am Mineral 92:481-490. doi:10.2138/am.2007.2305

Barin I (1989) Thermochemical data of pure substances, part II. VCH Verlags-gesellschaft mbH, Weinheim

Besson JM, Pinceaux JP, Anastopolous C, Velde B (1982) Raman spectra of olivine up to 65 kilobars. J Geophys Res 87:1077310775. doi:10.1029/JB087iB13p10773

Burns RG (1985) Thermodynamic data from crystal field spectra. Microscopic to macroscopic-atomic environments to mineral thermodynamics. Rev Mineral 14:277-316

Burns RG, Sung CM (1978) The effect of crystal field stabilization on the olivine-spinel transition in the system $\mathrm{Mg}_{2} \mathrm{SiO}_{4}-\mathrm{Fe}_{2} \mathrm{SiO}_{4}$. Phys Chem Miner 2:349-364. doi:10.1007/BF00307577

Chaplot SL, Choudhury N, Ghose S, Rao MN, Mittal R, Goel P (2002) Inelastic neutron scattering and lattice dynamics of 
minerals. Eur J Mineral 14:291-329. doi:10.1127/0935-1221/ 2002/0014-0291

Chase MW Jr, Davies CA, Downey JR Jr, Frurip DJ, McDonald RA, Syverud AN (1985) J Phys Chem Ref Data Suppl 1, 14, pp 1469 and 1489

Chopelas A (1990) Thermal properties of forsterite at mantle pressures derived from vibrational spectroscopy. Phys Chem Miner 17:149-156

Chopelas A (1991) Single-crystal spectra of forsterite, fayalite, and monticellite. Am Mineral 76:1101-1109

Chopelas A (1999) Estimates of mantle relevant Clapeyron slopes in the $\mathrm{MgSiO}_{3}$ system from high-pressure spectroscopic data. Am Mineral 84:233-244

Chopelas A (2000) Thermal expansivity of mantle relevant magnesium silicates derived from vibrational spectroscopy at high pressure. Am Mineral 85:270-278

Dachs E, Geiger CA (2007) Entropies of mixing and subsolidus phase relation of forsterite-fayalite $\left(\mathrm{Mg}_{2} \mathrm{SiO}_{4}-\mathrm{Fe}_{2} \mathrm{SiO}_{4}\right)$ solid solution. Am Mineral 92:699-702. doi:10.2138/am.2007.2465

Dachs E, Geiger CA, von Seckendorff V, Grodzicki M (2007) A lowtemperature calorimetric study of sysnthetic (forsterite + fayalite) $\mathrm{Mg}_{2} \mathrm{SiO}_{4}+\mathrm{Fe}_{2} \mathrm{SiO}_{4}$ solid solutions: an analysis of vibrational, magnetic and electronic contributions to the molar heat capacity and entropy of mixing. J Chem Thermodyn 39:906-933. doi:10.1016/j.jct.2006.11.009

Darling KL, Gwanmesia GD, King J, Li B, Liebermann RC (2004) Ultrasonic measurements of the sound velocities in polycrystalline San Carlos olivine in multi-anvil, high-pressure apparatus. Phys Earth Planet Inter 143-144:19-31. doi:10.1016/j.pepi.2003. 07.018

Deuss A, Redfern SAT, Chambers K, Woodhouse JH (2006) The nature of the 660-kilometer discontinuity in Earth's mantle from global seismic observations of PP precursors. Science 311:198201. doi:10.1126/science. 1120020

Dinsdale AT (1991) SGTE data for pure elements. Calphad 15:317425. doi:10.1016/0364-5916(91)90030-N

Dziewonski AM, Anderson DL (1981) Preliminary reference Earth model. Phys Earth Planet Inter 25:297-356. doi:10.1016/00319201(81)90046-7

Esser H, Averdieck R, Grass W (1933) Wärmeinhalt einiger metalle, legierungen und schlackenbildner bei temperaturen bis $1200^{\circ}$. Arch Eisenhuttenwesen 6:289-292

Fabrichnaya O (1998) The assessment of thermodynamic parameters for solid phases in the $\mathrm{Fe}-\mathrm{Mg}-\mathrm{O}$ and $\mathrm{Fe}-\mathrm{Mg}-\mathrm{Si}-\mathrm{O}$ systems. Calphad 22:85-125

Fisher GW, Medaris LG (1969) Cell dimensions and X-ray determinative curve for synthetic Mg-Fe olivines. Am Mineral 54:741753

Gillet P, Richet P, Guyot F, Fiquet G (1991) High-temperature thermodynamic properties of forsterite. J Geophys Res 96:1180511816. doi:10.1029/91JB00680

Gillet P, Daniel I, Guyot F, Matas J, Chervin J-C (2000) A thermodynamic model for $\mathrm{MgSiO}_{3}$-perovskite derived from pressure, temperature and volume dependence of the Raman mode frequencies. Phys Earth Planet Inter 117:361-384. doi: 10.1016/S0031-9201(99)00107-7

Graham EK, Schwab JA, Sopkin SM, Takei H (1988) The pressure and temperature dependence of the elastic properties of singlecrystal fayalite $\mathrm{Fe}_{2} \mathrm{SiO}_{4}$. Phys Chem Miner 16:186-198. doi: 10.1007/BF00203203

Guyot F, Boyer H, Madon M, Velde B, Poirier JP (1986) Comparison of the Raman microprobe spectra of $(\mathrm{Mg}, \mathrm{Fe})_{2} \mathrm{SiO}_{4}$ and $\mathrm{Mg}_{2} \mathrm{GeO}_{4}$ with olivine and spinel structures. Phys Chem Miner 13:91-95. doi:10.1007/BF00311898

Hayashi M, Tamura I, Shimomura O, Sawamoto H, Kawamura H (1987) Antiferromagnetic transition of fayalite under high pressure studied by Mössbauer spectroscopy. Phys Chem Miner 14:341-344. doi:10.1007/BF00309807

Hazen RM (1977) Effects of temperature and pressure on the crystal structure of ferromagnesian olivine. Am Mineral 62:286-295

Hofmeister AM (1987) Single-crystal absorption and reflection infrared spectroscopy of forsterite and fayalite. Phys Chem Miner 14:499-513. doi:10.1007/BF00308285

Hofmeister AM, Ito E (1992) Thermodynamic properties of $\mathrm{MgSiO}_{3}$ ilmenite from vibrational spectra. Phys Chem Miner 18:423432. doi:10.1007/BF00200965

Hofmeister AM, Mao H-K (2001) Evaluation of shear moduli and other properties of silicates with the spinel structure from IR spectroscopy. Am Mineral 86:622-639

Hofmeister AM, Xu J, Mao H-K, Bell PM, Hoering TC (1989) Thermodynamics of $\mathrm{Fe}-\mathrm{Mg}$ olivines at mantle pressures: midand far-infrared spectroscopy at high pressure. Am Mineral 74:281-306

Holland T, Powell R (1998) An internally consistent thermodynamic dataset for phases of petrological interest. J Metamorph Geol 16:309-343. doi:10.1111/j.1525-1314.1998.00140.x

Huang E, Chen CH, Huang T, Lin EH, Xu J-A (2000) Raman spectroscopic characteristics of $\mathrm{Mg}-\mathrm{Fe}-\mathrm{Ca}$ pyroxenes. $\mathrm{Am}$ Mineral 85:473-479

Huggins FE (1974) Mössbauer studies of iron minerals under pressure of up to 200 kilobars. Ph.D thesis, MIT

Isaak DG (1992) High-temperature elasticity of iron-bearing olivines. J Geophys Res 97:1871-1885. doi:10.1029/91JB02675

Isaak DG, Graham EK, Bass JD, Wang H (1993) The elastic properties of single-crystal fayalite as determined by dynamical measurement techniques. Pure Appl Geophys 141:393-414. doi: 10.1007/BF00998337

Jackson I, Webb S, Weston L, Boness D (2005) Frequency dependence of elastic wave speeds at high temperature: a direct experimental demonstration. Phys Earth Planet Inter 148:85-96. doi:10.1016/j.pepi.2004.08.004

Jacobs MHG, de Jong BHWS, Oonk HAJ (2001) The Gibbs energy formulation of $\alpha, \gamma$ and liquid $\mathrm{Fe}_{2} \mathrm{SiO}_{4}$ using Grover, Getting and Kennedy's empirical relation between volume and bulk modulus. Geochim Cosmochim Acta 65:4231-4242. doi:10.1016/ S0016-7037(01)00694-9

Jacobs MHG, de Jong BHWS (2005a) Quantum-thermodynamic treatment of intrinsic anharmonicity; Wallace's theorem revisited. Phys Chem Miner 32:614-626. doi:10.1007/s00269-0050038-x

Jacobs MHG, de Jong BHWS (2005b) An investigation into thermodynamic consistency of data for the olivine, wadsleyite and ringwoodite for of $(\mathrm{Mg}, \mathrm{Fe})_{2} \mathrm{SiO}_{4}$. Geochim Cosmochim Acta 69:4361-4375. doi:10.1016/j.gca.2005.05.002

Jacobs MHG, van den Berg AP, de Jong BHWS (2006) The derivation of thermo-physical properties and phase equilibria of silicate materials from lattice vibrations: application to convection in the Earth's mantle. Calphad 30:131-146. doi:10.1016/ j.calphad.2005.10.001

Jacobs MHG, de Jong BHWS (2007) Putting constraints on phase equilibria and thermo-physical properties in the system $\mathrm{MgO}$ $\mathrm{SiO}_{2}$ by a thermodynamically consistent vibrational method. Geochim Cosmochim Acta 71:3630-3655. doi:10.1016/j.gca. 2007.05.010

Jeanloz R, Thompson AB (1983) Phase transitions and mantle discontinuities. Rev Geophys Space Phys 21:51-74. doi: 10.1029/RG021i001p00051

Kelley KK (1943) Specific heats at low temperatures of magnesium orthosilicate and magnesium metasilicate. J Am Chem Soc 65:339-341. doi:10.1021/ja01243a012

Kieffer SW (1979) Thermodynamics and lattice vibrations of minerals: 3 lattice dynamics and an approximation for minerals 
with application to simple substances and framework silicates. Rev Geophys Space Phys 17:35-59. doi:10.1029/RG017i001 p00035

King EG (1957) Low temperature heat capacities and entropies at $298.15 \mathrm{~K}$ of some crystalline silicates containing calcium. J Am Chem Soc 79:5437-5438. doi:10.1021/ja01577a028

Kitayama K, Katsura K (1968) Activity measurements in orthosilicate, metasilicate solid solutions- $\mathrm{I} . \mathrm{Mg}_{2} \mathrm{SiO}_{4}-\mathrm{Fe}_{2} \mathrm{SiO}_{4}, \mathrm{MgSiO}_{3}$ $\mathrm{FeSiO}_{3}$ at $1204^{\circ} \mathrm{c}$. Bull Chem Soc Jpn 41:1146-1151. doi: $10.1246 / \mathrm{bcsj} .41 .1146$

Kojitani H, Akaogi M (1994) Calorimetric study of olivine solid solutions in the system $\mathrm{Mg}_{2} \mathrm{SiO}_{4}-\mathrm{Fe}_{2} \mathrm{SiO}_{4}$. Phys Chem Miner 20:536-540. doi:10.1007/BF00211849

Kudoh Y, Takeda H (1986) Single crystal X-ray diffraction study on the bond compressibility of fayalite, $\mathrm{Fe}_{2} \mathrm{SiO}_{4}$ and rutile, $\mathrm{TiO}_{2}$ under high pressure. Physica 139 and 140B:333-336

Lawson AW (1947) On simple binary solid solutions. J Chem Phys 15:831-842. doi:10.1063/1.1746346

Liu W, Li B (2006) Thermal equation of state of $\left(\mathrm{Mg}_{0.9} \mathrm{Fe}_{0.1}\right)_{2} \mathrm{SiO}_{4}$ olivine. Phys Earth Planet Inter 157:188-195. doi:10.1016/ j.pepi.2006.04.003

Mercier JC, Carter NL (1975) Pyroxene geotherms. J Geophys Res 80:3349-3362. doi:10.1029/JB080i023p03349

Mernagh TP, Hoatson DM (1997) Raman spectroscopic study of pyroxene structures from the Munni Munni layered intrusion, Western Australia. J Raman Spectrosc 28:647-658. doi:10.1002/ (SICI)1097-4555(199709)28:9<647::AID-JRS155>3.0.CO;2-H

Nafziger RH, Muan A (1967) Equilibrium phase compositions and themodynamic properties of olivines and pyroxenes in the system $\mathrm{MgO}-$ "FeO"- $\mathrm{SiO}_{2}$. Am Mineral 52:1364-1385

Orr RL (1953) High temperature heat contents of magnesium orthosilicate and ferrous orthosilicate. J Am Chem Soc 75:528-529. doi:10.1021/ja01099a005

Plymate TG, Stout JH (1990) Pressure-volume-temperature behavior of fayalite based on static compression measurements at $400^{\circ} \mathrm{C}$. Phys Chem Miner 17:212-219. doi:10.1007/BF00201452

Reynard B, Rubie DC (1996) High-pressure, high-temperature Raman spectroscopic study of ilmenite-type $\mathrm{MgSiO}_{3}$. Am Mineral 81:1092-1096

Richard G, Richet P (1990) Room temperature amorphization of fayalite and high pressure properties of $\mathrm{Fe}_{2} \mathrm{SiO}_{4}$ liquid. Geophys Res Lett 17:2093-2096. doi:10.1029/GL017i012p02093

Robie RA, Finch CB, Hemingway BS (1982) Heat capacity and entropy of fayalite $\left(\mathrm{Fe}_{2} \mathrm{SiO}_{4}\right)$ between 5.1 and $383 \mathrm{~K}$ : comparison of calorimetric and equilibrium values of the QFM buffer reaction. Am Mineral 67:463-469

Sack RO, Ghiorso MS (1989) Importance of considerations of mixing properties in establishing an internally consistent thermodynamic database: thermochemistry of minerals in the system $\mathrm{Mg}_{2} \mathrm{SiO}_{4}$ $\mathrm{Fe}_{2} \mathrm{SiO}_{4}-\mathrm{SiO}_{2}$. Contrib Mineral Petrol 102:41-68. doi:10.1007/ BF01160190

Saxena SK (1996) Earth mineralogical model: Gibbs free energy minimization computation in the system $\mathrm{MgO}-\mathrm{FeO}-\mathrm{SiO} 2$. Geochim Cosmochim Acta 60:2379-2395. doi:10.1016/00167037(96)00096-8

Schwab von RG, Küstner D (1977) Präzisionsgitterkonstantenbestimming zur festlegung röntgenographischer bestimmungskurven für synthetische olivine der mischkristallreihe forsterit-fayalite. Neues Jahrbuch für Mineralogie. MH:205-215

Smyth JR (1975) High temperature crystal chemistry of fayalite. Am Mineral 60:1092-1097
Sumino Y (1979) The elastic constants of $\mathrm{Mn}_{2} \mathrm{SiO}_{4}, \mathrm{Fe}_{2} \mathrm{SiO}_{4}$ and $\mathrm{Co}_{2} \mathrm{SiO}_{4}$, and the elastic properties of olivine group minerals at high temperature. J Phys Earth 27:209-238

Suzuki I, Seya K, Takei H, Sumino Y (1981) Thermal expansion of fayalite $\left(\mathrm{Fe}_{2} \mathrm{SiO}_{4}\right)$. Phys Chem Miner 7:60-63. doi:10.1007/ BF00309452

Suzuki I, Anderson OL, Sumino Y (1983) Elastic properties of a single-crystal forsterite $\mathrm{Mg}_{2} \mathrm{SiO}_{4}$ up to $1200 \mathrm{~K}$. Phys Chem Miner 10:38-46. doi:10.1007/BF01204324

Takahashi $\mathrm{T}$ (1970) Isothermal compression of fayalite at room temperature (abs). Eos Trans AGU 51:827

Vinet P, Ferrante J, Rose JH, Smith JR (1987) Compressibility of solids. J Geophys Res 92:9319-9325. doi:10.1029/JB092iB09 p09319

Wallace DC (1972) Thermodynamics of crystals. Wiley, New York

Wang H, Bass JD, Rossman GR (1989) Elastic properties of Febearing pyroxenes and olivines. Eos Trans AGU 40:474

Wang SY, Sharma SK, Cooney TF (1992) Micro-Raman and infrared spectral study of forsterite under high pressure. Am Mineral 78:469-476

Watanabe H (1982) Thermochemical properties of synthetic highpressure compounds relevant to the earth's mantle. Highpressure research in geophysics. In: Manghnani MH, Akimoto S (eds) Center for Academic Publications, Japan, pp 441-464

Watanabe $\mathrm{H}$ (1987) Physico-chemical properties of olivine and spinel solid solutions in the system $\mathrm{Mg}_{2} \mathrm{SiO}_{4}-\mathrm{Fe}_{2} \mathrm{SiO}_{4}$. In: Manghnani $\mathrm{MH}$, Syono $\mathrm{Y}$ (eds) High-pressure research in mineral physics. Terra Scientific Publishing Company (TERRAPUB), Tokyo/ AGU, Washington DC, pp 275-278

Webb SL, Jackson I, Takei H (1984) On the absence of shear mode softening in single-crystal fayalite $\mathrm{Fe}_{2} \mathrm{SiO}_{4}$ at high pressure and room temperature. Phys Chem Miner 11:167-171. doi:10.1007/ BF00387847

Williams Q, Knittle E, Reichlin R, Martin S, Jeanloz R (1990) Structural and electronic properties of $\mathrm{Fe}_{2} \mathrm{SiO}_{4}$-fayalite at ultrahigh pressures: amorphization and gap closure. J Geophys Res 95:21549-21563. doi:10.1029/JB095iB13p21549

Wiser NM, Wood BJ (1991) Experimental determination of activities in $\mathrm{Fe}-\mathrm{Mg}$ olivine at $1400 \mathrm{~K}$. Contrib Mineral Petrol 108:146153. doi:10.1007/BF00307333

Wood BJ, Kleppa OJ (1981) Thermochemistry of forsterite-fayalite olivine solution. Geochim Cosmochim Acta 45:529-534. doi: 10.1016/0016-7037(81)90185-X

Yagi T, Ida Y, Sato Y, Akimoto S (1975) Effect of hydrostatic pressure on the lattice parameters of $\mathrm{Fe}_{2} \mathrm{SiO}_{4}$ olivine up to 70 kbar. Phys Earth Planet Inter 10:348-354. doi:10.1016/00319201(75)90062-X

Yagi T, Akaogi M, Shimomura O, Suzuki T, Akimoto S (1987) In situ observation of the olivine-spinel phase transformation in $\mathrm{Fe}_{2} \mathrm{SiO}_{4}$ using synchrotron radiation. J Geophys Res 92:62076213. doi:10.1029/JB092iB07p06207

Zaug JM, Abramson EH, Brown JM, Slutsky LJ (1993) Sound velocities in olivine at earth mantle pressures. Science 260:1487-1489. doi:10.1126/science.260.5113.1487

Zha C-S, Duffy TS, Downs RT, Mao H-K, Hemley RJ (1998) Brillouin scattering and X-ray diffraction of San Carlos olivine: direct pressure determination to $32 \mathrm{GPa}$. Earth Planet Sci Lett 159:25-33. doi:10.1016/S0012-821X(98)00063-6

Zhang L (1998) Single crystal hydrostatic compression of (Mg, Mn, $\mathrm{Fe}, \mathrm{Co})_{2} \mathrm{SiO}_{4}$ olivines. Phys Chem Minerals 25:308-312 